\title{
Ein Bergrat, zwei Minister und sechs Lehrende
}

\author{
Versuche der Gründung einer Bergakademie in Berlin um \\ 1770
}

Ursula Klein

A Mining Councillor, Two Ministers, and Six Tutors. Attempts to Establish a Mining School in Berlin around 1770

After the Seven Years War, the Prussian administration launched a campaign for useful knowledge and scientific education of state officials. This essay scrutinizes efforts undertaken around 1770 by the Central Prussian Administration for Mining and Smelting Works to establish the "mining sciences" and a mining school in the city of Berlin. As a result, from October 1770 on this Administration supported the public teaching in Berlin of mathematics, mechanics, mineralogy, metallurgy, chemistry and other areas of knowledge that constituted the "mining sciences." In so doing, it promoted the interconnection of scientific and technological knowledge. Contrary to common belief, however, a mining school or academy was not established in eighteenth-century Berlin.

Keywords: useful knowledge, Berlin Mining Academy, cameralists, scientific-technological experts, Carl Abraham Gerhard

Schlüsse/worter: Nützliches Wissen, Berliner Bergakademie, Kameralisten, wissenschaftlich-technische Experten, Carl Abraham Gerhard

Im Vorfrühling 1770, zu einer Jahreszeit als man längere Reisen noch tunlichst vermied und mehr als einen Monat vor Beginn seiner jährlichen Inspektionsreisen in die preußischen Bergbauregionen, fuhr der preußische Bergrat Carl Abraham Gerhard (1738-1821) von Berlin in die sächsische Bergstadt Freiberg. Dort sollte er im Auftrag des preußischen Bergwerks- und Hüttendepartments Erkundigungen über die fünf Jahre zuvor errichtete Freiberger Bergakademie einholen. Mehrere Minister in der zentralen preußischen Behörde, dem „Generaldirektorium“, überlegten schon seit längerem wie man die Arbeit der preußischen Beamten verbessern könnte. ${ }^{1}$ Fachleute waren gesucht, die Sachkenntnisse über das Berg- und Hüttenwesen und andere Gewerbe besaßen. ${ }^{2}$

Im Mai 1768 war das Bergwerks- und Hüttendepartment in Berlin als zentrale Fachbehörde für das gesamte preußische Bergbau- und Hüttenwesen 
gegründet worden, um dieselbe Zeit wurden die lokalen Oberberg- und Bergämter in den preußischen Provinzen reorganisiert. Dem folgte die Gründung eines Forstdepartments im Januar 1770 und eines Oberbaudepartments im Juni desselben Jahres. Somit schienen geeignete Instrumente für die Wirtschaftsförderung und Steigerung der Staatseinkünfte vorhanden zu sein, doch auf allen Ebenen fehlten kompetente Experten. Dem sollte durch eine verbesserte wissenschaftliche Ausbildung der preußischen Beamten Abhilfe geschaffen werden. Um 1770 initiierten die preußischen Behörden eine noch nie da gewesene Kampagne für die Lehre sogenannter nützlicher Wissenschaften. Das Studium der Mathematik, Chemie, Physik, Mineralogie, Geologie und anderer Teile der „Bergwerkswissenschaften“, der Botanik und Forstwissenschaft, der Staats- und Kameralwissenschaft wurde mit großem Nachdruck propagiert. Die Zeit der „Idioten und Ignoranten“ im preußischen Staatsdienst sollte endlich ein Ende haben, so der Leiter des Bergwerks- und Hüttendepartments, Minister Ludwig Philipp Freiherr von Hagen (1724-1771). ${ }^{3}$

Am 24. März 1770, kurz nach seiner Rückkehr aus Freiberg legte Gerhard Minister von Hagen, seinen Bericht über die Freiberger Bergakademie vor. Unmittelbar danach leitete von Hagen Aktivitäten für die wissenschaftliche und technologische Ausbildung der preußischen Berg- und Hüttenbeamten ein, die deutlich vom Vorbild der Freiberger Bergakademie abwichen. Aber was hatte man von dieser Reise erwartet? Welche Überlegungen gab es in der Residenzstadt Berlin über nützliche Wissenschaften und die Reform der Ausbildung der Berg- und Hüttenbeamten?

Im Laufe des Jahres 1770 unternahmen leitende Beamte der preußischen Behörden ein ganzes Bündel konkreter Maßnahmen für die Förderung der Lehre nützlicher Wissenschaften, die von Verordnungen für die Universitäten und Realschulen bis zum Versuch der Errichtung einer Bergschule in Berlin reichten. Die einzelnen Reformmaßnahmen wurden von Vorschlägen und Diskussionen über die einzuschlagenden Wege und institutionellen Alternativen begleitet, die die Entschlossenheit der beteiligten Akteure bezeugen, eine wissenschaftliche Reform der Beamtenausbildung herbeizuführen. Im Folgenden wird ein Einblick in einen Teil dieser Reformmaßnahmen gegeben, und zwar in die Einführung öffentlichen Unterrichts nützlicher Wissenschaften durch das Berliner Bergwerks- und Hüttendepartment.

Eine dichte Rekonstruktion dieser lokalen Aktivitäten ist aus mehreren Gründen ein Desiderat. Zum einen bedarf die Frage, was die historischen Akteure unter nützlichen Wissenschaften verstanden einer genauen epistemologischen Analyse, die auch der Vielfalt der Wissenschaften und Auffassungen gerecht wird. Eine solche Analyse wird hier vorgelegt, und zwar begrenzt auf die Auffassungen der lokalen Akteure. Zum anderen sind für ein adäquates historisches Verständnis der Erfolge und Misserfolge der kameralistischen Versuche, Wissenschaften und technologische Innovation miteinander zu verknüpfen nach wie vor nicht genügend detaillierte Fallstudien 
vorhanden, insbesondere solche, in denen das Augenmerk nicht nur auf die Rhetorik, sondern auch auf die Praxis der involvierten Akteure gelegt wird. Dabei sind auch die Machkonstellationen und Gestaltungsspielräume der Kameralisten im absolutistischen Staat zu berücksichtigen. In der folgenden Rekonstruktion geht es daher auch um die Frage, welche Personen - Individuen und soziale Figuren - aktiv an der Ausbildungsreform preußischer Bergund Hüttenbeamten beteiligt waren. So ist beispielsweise behauptet worden, der preußische König Friedrich II. sei als „Philosophenkönig“ und Liebhaber der Wissenschaften an einer wissenschaftlichen Ausbildung seiner Beamten interessiert gewesen und habe diese gefördert (Krusch 1904, Kahlow 2000). Dafür lassen sich jedoch in den Dokumenten keine Anhaltspunkte finden. Jedenfalls war das Interesse Friedrichs II. nicht so stark, dass er auch nur einen winzigen Bruchteil der Gelder, die in seine Armee flossen, für eine solche Reform zur Verfügung gestellt hätte. ${ }^{4}$ Vielmehr zeigt sich, dass die Bemühungen preußischer Kameralisten für eine Reform der Beamtenausbildung durch die Finanzpolitik Friedrichs II. unterlaufen wurden.

Ein Hauptanliegen des vorliegenden Aufsatzes ist eine genaue historische Rekonstruktion der Aktivitäten der preußischen Behörden um 1770 für eine Reform der Ausbildung von Berg- und Hüttenbeamten. Diese Rekonstruktion wird zeigen, dass die in der historischen Literatur fast einhellig vertretene Auffassung, in Berlin habe es ab 1770 eine Bergakademie gegeben, revidiert werden muss. ${ }^{5}$ Die Idee der Gründung einer Bergschule oder -akademie wurde in der Residenzstadt Berlin anfangs zwar diskutiert, dann aber schnell wieder fallen gelassen. Statt dessen organisierte und finanzierte das Bergwerks- und Hüttendepartment eine öffentliche Vorlesungsreihe über nützliche Wissenschaften, die sich sowohl an künftige Berg- und Hüttenbeamte als auch an Beamte für das Bau- und Forstwesen wandte und die darüber hinaus auch von Medizinern und Apothekern besucht wurde. Diese Vorlesungen wurden nach 1774 gelegentlich durch Laborunterricht, praktischen Zeichenunterricht und Besuche des Mineralienkabinetts des Bergwerks- und Hüttendepartments ergänzt. Eine Bergakademie wurde in Berlin jedoch vor 1860 nicht etabliert. Bereits 1776 gab es die ersten alternativen Lehrangebote für Baubeamte, die mit der Reaktivierung der Akademie der Künste und der 1799 gegründeten Bauakademie eigenständige Lehranstalten erhielten (Kahlow 2000, Lundgren 1975). Wohlhabende Anwärter für die preußischen Bergbehörden studierten dagegen auch in den Folgejahrzehnten nicht selten an der Freiberger Bergakademie.

\section{Bergrat Gerhard: eine Bergschule für Berlin}

Carl Abraham Gerhard war seit 1768 Bergrat im neu gegründeten Bergwerksund Hüttendepartment in Berlin. Er hatte 1755 am Berliner Collegium 
medico-chirurgicum studiert und dann sein Medizinstudium an der Universität Frankfurt/Oder fortgesetzt, wo er 1760 promovierte. ${ }^{6}$ Als Bergrat in der zentralen preußischen Behörde für das Bergwerks- und Hüttenwesen hatte er ein umfangreiches Aufgabenfeld vor sich, das von jährlichen Inspektionsreisen in die preußischen Montanregionen, über die Vorbereitung administrativer Maßnahmen seines vorgesetzten Ministers in der Berliner Behörde bis hin zu technischen Verbesserungen und Innovationsprojekten und der Organisation der Ausbildungsreform der preußischen Berg- und Hüttenbeamten reichte. Ab 1770 war Gerhard auch selbst Dozent in der vom ihm organisierten Vorlesungsreihe des Berliner Bergwerks- und Hüttendepartments, die als "Bergschule“ oder „Bergakademie“ in die Geschichte eingegangen ist. Zugleich war er Mitglied der Preußischen Akademie der Wissenschaften und ein angesehener Naturforscher, der durch seine mineralogischen, geognostischen und chemischen Publikationen in der Gelehrtenwelt bekannt war. ${ }^{7}$

Gerhard verkörperte geradezu das Ideal des ,guten', mit administrativen, wissenschaftlichen und technischen Sachkenntnissen gerüsteten Kameralisten. Seine Interessen und Aktivitäten gingen aber nicht vollständig in der Agenda eines Kameralisten auf, wie dies beispielsweise bei seinem späteren Vorgesetzten, dem preußischen Minister Friedrich Anton von Heynitz der Fall war. Gerhard gehörte zur ersten Generation sachkompetenter, wissenschaftlich gebildeter Kameralisten in Preußen, die selbst noch keine Lehre oder schulische Ausbildung für ihre technischen Beamtenfunktionen absolviert hatten. Er war ein angesehener Naturforscher, hauptsächlich Mineraloge, der am gelehrten Naturdiskurs partizipierte und sich nach und nach auch zum technischen Experten entwickelte. ${ }^{8}$ Gerhard produzierte mineralogisches, geologisches und chemisches Wissen in engem Zusammenhang mit seinen praktischen, technischen Aufgaben als Bergrat, seinen Versuchen der Verbesserung von Gruben und Hüttenwerken und seinem Unterricht in der Berliner „Bergschule“. Solche hybriden wissenschaftlich-technischen Experten, die Naturforschung und technische Praxis unter einen Hut brachten, waren besonders zahlreich unter den Lehrern der Bergakademien und ähnlicher technischer Fachhochschulen des 18. und frühen 19. Jahrhunderts vertreten. ${ }^{9}$ Durch die Ausbildung technisch kompetenter Beamter leisteten sie einen Beitrag zur Verwaltungsreform und Industrialisierung. Gleichzeitig bereicherten sie eine Wissenstradition, die beobachtende und experimentelle Naturforschung mit Objekten der Technik und staatlichen Innovationsprojekten vernetzte.

Die Figur des wissenschaftlich-technischen Experten - der Naturforscher und oft auch Lehrer nützlicher Wissenschaften war und gleichzeitig technischen Aufgaben nachging - existierte nicht nur im Berg- und Hüttenwesen. Es handelt sich dabei um ein allgemeineres sozio-kulturelles Phänomen der absolutistisch-merkantilistischen Staaten, das bisher nur unzureichend historisch untersucht wurde. ${ }^{10}$ Man trifft sie nicht nur in Behörden und unter 
Kameralisten, sondern auch in wissenschaftlichen Gesellschaften und Akademien, im Berliner Collegium medico-chirurgicum und privaten, chemisch-pharmazeutischen Lehrinstituten, in Laboratorien von Apothekern, Porzellanmanufakturen und der Artillerie, in botanischen Gärten und forstwirtschaftlichen Plantagen, in Werkstätten von Instrumentenbauern und Mathematikern, in der Königlichen Gewerbeakademie des frühen 19. Jahrhunderts. Jedoch in keiner preußischen Institution wurde sie so gezielt gefördert wie in den neuen sachbezogenen Behörden, die nach dem Siebenjährigen Krieg allmählich entstanden und nach den Napoleonischen Kriegen mit den SteinHardenbergschen Reformen fest verankert wurden. Minister und andere hohe Kameralisten dieser neuen Fachbehörden betrieben eine aktive Rekrutierungspolitik für wissenschaftlich gebildete, technische Beamte, und sie boten höhere Beamtenpositionen nicht selten solchen Experten an, die wie Gerhard gleichzeitig auch als Naturforscher tätig und bekannt waren.

Anfang Januar 1770 erhielt Gerhard von Minister von Hagen den Auftrag, einen Plan zur Errichtung einer Bergschule in Berlin zu erarbeiten. Die ersten Bergschulen oder -akademien, die vermutlich dazu die Anregung gaben, waren 1765 in der sächsischen Bergstadt Freiberg und fast zeitgleich im österreichisch-ungarischen Schemnitz gegründet worden (Brianta 2000). Am 23. Januar 1770 legte Gerhard auftragsgemäß seinen Plan zu einer solchen „volständigen Berg-Information“ vor. ${ }^{11}$ Es handelte sich dabei um inhaltliche und organisatorische Vorschläge für eine in Berlin $\mathrm{zu}$ errichtende höhere Schule, die künftigen preußischen Berg- und Hüttenbeamten eine wissenschaftliche Grundausbildung geben sollte. Auch ein Laboratorium und eine Modellsammlung waren vorgesehen. ${ }^{12}$

Der Grundgedanke von Gerhards Plan war, allen Berg- und Hüttenbeamten Preußens ein gemeinsames wissenschaftliches Fundament zu geben, das für ihre späteren Amtsfunktionen nützlich sein konnte. Die „Theorie“ das heißt in diesem Kontext Schulwissen und - unterricht - sollte vor der praktischen Lehre in Gruben und auf Hüttenwerken, kommen. Ein Berg- oder Hüttenmann sollte ,zuförderst mit denen nöthigen theoretisch[en] Kenntnissen versehen seyn, ehe er sich mit Nutzen an die practische Ausübung dieses Metiers" machte. ${ }^{13}$ Die ,jungen Leute“ im Alter von fünfzehn bis sechzehn Jahren sollten zuerst das „Metier [...] systematisch und scientifisch erlernen“; und erst „wenn dergleichen Eleves mit diesen Principiis versehen“ waren, sollten sie noch „ein Jahr auf Berg-Wercke“ gehen. ${ }^{14}$ Die wissenschaftliche Ausbildung für alle zukünftigen Berg- und Hüttenbeamten an der Berliner Bergschule sollte nach Gerhards Vorstellungen zweieinhalb Jahre dauern. Im Anschluss daran war ein Jahr Praxis in den Gruben oder Hüttenwerken der preußischen Provinzen vorgesehen. Daher differenzierte Gerhard auch nicht zwischen höheren Berg- und Hüttenbeamten, die für Leitungsfunktionen im Bergwerks- und Hüttendepartment in Berlin oder den Oberbergämtern der preußischen Provinzen vorgesehen waren, und 


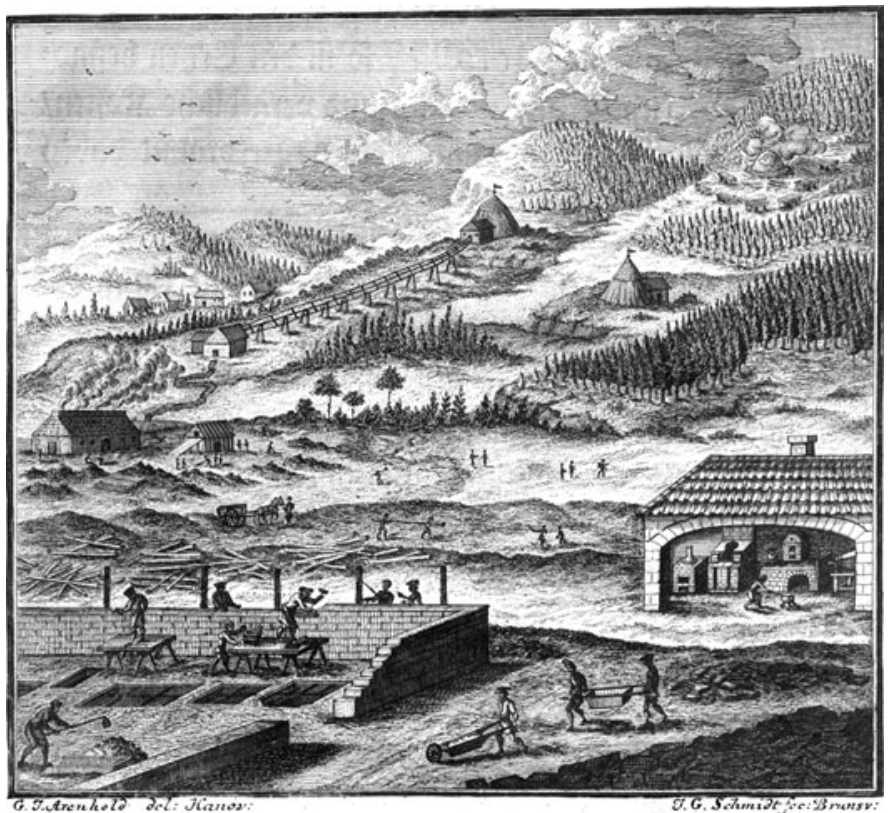

Abb. 1 Kombiniertes Berg- und Hüttenwerk. (Aus: Schlüter 1738: 0. S.)

niedrigeren Beamten, die spezielle technische Aufgaben in den Gruben und Hüttenwerken wahrnehmen sollten. „Ein jeder, so sich dem Berg- und HüttenWesen widmen will“" und jeder, der in Preußen dieses „Metier ausüben will“, so Gerhard, sollte diese schulische Bildung erhalten. ${ }^{15}$ Diese sollte gleichsam das Fundament für alle nachfolgenden Spezialisierungen abgeben.

Die Bergschule, die Gerhard im Auge hatte, diente also nicht der speziellen beruflichen Ausbildung für besondere Beamtenpositionen, sondern der Hebung des Bildungsniveaus aller preußischer Berg- und Hüttenbeamten. Der Plan passte in damalige Reformbestrebungen, die mit der Etablierung von „Bergwerkswissenschaften“ eine allgemeine Förderung des Berg- und Hüttenwesens zum Nutzen des Staates und des Gemeinwohls anstrebten. Dies geht nicht zuletzt aus zahlreichen Übereinstimmungen hervor, die zwischen Gerhards Plan und einem früheren Vorschlag Carl Friedrich Zimmermanns für eine sächsische Bergschule bestehen (Zimmermann 1769). Allerdings gab es im Rahmen dieser Reformbestrebungen unterschiedliche Vorstellungen über das Verhältnis von schulischer Ausbildung und Praxis. An der Bergakademie Freiberg waren Vorlesungen - also die schulische oder „theoretische“ Ausbildung - verknüpft mit der praktischen Ausbildung durch Grubenbefahrung, Besuch von Hüttenwerken, Zeichenlehre, Markscheider- und chemische Probierlehre. Gerhards radikale Vorstellung einer zeitlichen Trennung von Theorie und Praxis war zwar für die Residenzstadt Berlin praktikabel, stieß jedoch auf mächtigen Widerspruch, wie ich im Folgenden zeigen werde. 


\section{Nützliche Bergwerkswissenschaften}

Was verstanden Gerhard und seine Zeitgenossen unter nützlichen Wissenschaften und unter Bergwerkswissenschaften? Eine Analyse der Lehrinhalte in Gerhards Plan gibt hierüber Auskunft. „Es gründet sich die Theorie des Bergbaus und Hüttenwesens", so fasste Gerhard sein Credo über nützliche Bergwerkswissenschaften zusammen, ,auf die Kenntniß der Mathematic, der Mineralogie, der Physik und den damit unzertrennlich verbundenen Mechanic und Hydraulik . ${ }^{16}$ "Theorie“ bedeutete hier nicht „wissenschaftliche Theorie“ im Sinne eines Wissenssystems, das klar von empirischem Wissen abgrenzbar ist, sondern in Schulen vermittelbares und vermitteltes Wissen, im Unterschied zur beruflichen Praxis. ${ }^{17}$ Die Mathematik ausgenommen, waren auch mit der Rede von „Prinzipien" und „allgemeinen Begriffen" keine Ausgangspunkte für Deduktionen und allgemeine Naturbegriffe gemeint, sondern systematisch gesammeltes und geordnetes taxonomisches und analytischchemisches Wissen. Gerhards Wissenschaftsverständnis und der kameralistische Diskurs des 17. und 18. Jahrhunderts über nützliche Wissenschaften entziehen sich einem adäquaten historisch-epistemologischen Verständnis, wenn Wissenschaft mit Theorie im Sinne von Newtons Principia oder der Materietheorien des 17. und 18. Jahrhunderts gleichgesetzt wird. Charakteristisch für den Wissenschaftsbegriff eines wissenschaftlich-technischen Experten und Kameralisten wie Gerhard ist vielmehr die Vermischung von Naturforschung und systematischem Wissen über die Natur mit technologischen Erkundungen und mathematisch-technischem oder Ingenieurswissen.

Gerhard wusste sehr wohl, dass die Mineralogie, ebenso wie andere Bereiche der Naturgeschichte und der Chemie, keine der newtonschen Mechanik vergleichbaren Theorien zu bieten hatte, die nutzbringend auf den Bergbau anwendbar gewesen wären. Anders verhielt es sich jedoch hinsichtlich der umfassenden, nicht an spezifische Lokalitäten gebundenen Materialienkenntnisse des Mineralogen und Chemikers und seiner Methoden, Materialien eindeutig $\mathrm{zu}$ identifizieren. Diese Art naturhistorischer und chemischer Kenntnisse und Fähigkeiten war nach Gerhards Überzeugung nützliches Wissen. „Ein jeder Berg- und Hütten-Mann muß doch wohl nothwendig die Ertze und Mineralien kennen, die er suchen, gewinnen und zu gute machen will", argumentierte er. Dies war vor allem deshalb notwendig, damit man nicht "wegen der besondern Mischung der Ertze fast an allen Orten" orientierungslos war und dann "lange und umsonst arbeiten“ musste. ${ }^{18}$ Der Mineraloge Gerhard meinte hier, dass man zuerst die Art oder Spezies eines Erzes oder Minerals kennen musste, bevor man, etwa bei der Erschließung einer neuen Lagerstätte, eine lokale Variante dieser Spezies identifizieren konnte. Es war ein epistemologisches Credo der Naturgeschichte des 18. Jahrhunderts, dass die Kenntnis der Charakteristika einer Spezies und der Techniken ihrer Identifikation auch die Identifikation der lokalen Varianten 
dieser Spezies erleichterte. Damit war eine Brücke zur Nützlichkeit der Naturgeschichte geschlagen. Ein gebildeter und geschickter Mineraloge konnte demnach eine lokal auftretende Variante beispielsweise von Bleiglanz ungleich verlässlicher als ein wertvolles Mineral identifizieren und über dessen Abbauwürdigkeit entscheiden als ein rein handwerklich ausgebildeter Bergmann, dessen Lehre im Rahmen lokaler Gegebenheiten erfolgte und der daher nur eine lokale Variante von Bleiglanz kennen und identifizieren gelernt hatte.

Mit ähnlichen Argumenten für translokales empirisches Wissen plädierte Gerhard auch für den frühen schulischen Erwerb von Kenntnissen der Geologie und der metallurgischen Chemie. Die handwerkliche, rein praktische Ausbildung, so Gerhard, hat zur Folge, dass

seine [des Berg- und Hüttenmanns, U.K.] ganze Erkenntnis nur auf einzelnen Erfahrung beruhet, die auf jedem Gebürge, auf jedem Hüttenwercke mannigfaltigen Veränderungen unterworfen seyn, welche zu entdecken wiederum erst neue Erfahrung erfordert werden, die gemeiniglich mit Verlust von Kosten und Zeit verbunden sind. ${ }^{19}$

Auch dieses Argument verband epistemologische und ökonomische Gesichtspunkte. Die systematische Kenntnis der Struktur der „Gebirge“, das heißt der Anordnungen der Gesteinsarten wie sie zum Beispiel von Gerhards Vorgänger Johann Gottlob Lehmann gelehrt wurde, ermöglichte demnach die zuverlässige Erkenntnis lokaler Gesteinsformationen in Gruben. Ebenso dienten die im chemischen Labor erworbenen empirischen Kenntnisse über das Schmelzverhalten der Metalle als „Leitfaden“ für ihr Schmelzverhalten bei der Verhüttung. „Ein sonst geschickter aber bloß practischer Hütten-Mann, der auf ein neues Werk kommt", so Gerhard, arbeitete daher weniger effektiv als ein chemisch gebildeter Schmelzer oder Probierer. ${ }^{20}$

Mathematik und Mechanik waren schon seit langem über die Empirie hinausgehende Wissensgebiete, die in der Markscheidekunst, dem Vermessen der Gruben unter und über Tage, und der Lehre der Kunstmeister vermittelt wurden. Mathematische Kenntnisse waren auch für das betriebliche Berechnungswesen in den Behörden notwendig. Gerhard erklärte daher den Nutzen von Mathematik und Physik folgendermaßen:

Da ferner bei dem Grubenbau Vermeßungen über und unter der Erde vorzunehmen sind, auch kein Berg- und Hütten-Werk sich ohne Maschinen die durch Feuer, oder Luft oder Wasser zu bewegen [sind], gedencken läßt, überdem so wohl bey dem Bergbau als auch bey dem Hütten-Wesen verschiedene Rechnungen vorkommen; so sind demselben [dem „Berg- und Hüttenmann] wohl die Mathematik nebst der Physic, Mechanic und Hydraulic, ganz unentbehrlich. ${ }^{21}$

In organisatorischer Hinsicht beinhalteten Gerhards Pläne für eine Bergschule genaue Festlegungen über die Abfolge der Vorlesungen während des zweieinhalbjährigen Studiums. Er sah also kein unverbindliches Angebot von Vorlesungen vor, aus dem frei gewählt werden konnte, wie dies faktisch später in Berlin 
der Fall war. Vielmehr hatte er auch hier genaue Vorstellungen wie zuerst Grundlagen geschaffen und dann auf diesen aufgebaut werden sollte. Im ersten Halbjahr sollte „Mathesis pura" und zusätzlich Mineralogie gelehrt werden; im zweiten Halbjahr Physik, einschließlich Mechanik und Hydraulik, und die Repetition der Mathematik; im dritten Halbjahr Chemie, einschließlich Metallurgie, und die Repetition der Physik; und schließlich aufbauend auf diesem Wissen im vierten und fünften Halbjahr „die Anweisung zum Grubenbau und Hüttenwesen “. ${ }^{22}$ Der für das letzte Jahr vorgesehene Unterricht war jedoch keine rein technische Instruktion, ebenso wie der vorangegangene Unterricht keine reine Naturlehre war. Er reichte vielmehr von der „Theorie von der Struktur der Erde“ über die „Lehre von denen Gebürgen“" (Gesteinskunde) bis zum Bau von Schächten und Stollen, Straßenbau, den Gewinnungs- und Transportmethoden der Erze, einschließlich der dazu notwenigen Instrumente und Maschinen, dem Pochen und Waschen der Erze, der nachfolgenden Identifikation der gereinigten Erze, dem Rösten der Erze und den dazu verwendeten Öfen, dem nachfolgenden Schmelzen der Erze, Schmelzöfen und Kohlen. Schließlich gehörte dazu auch die „Berg-Oeconomie“, die die Aufstellung von „Kosten Anschlägen“ lehrte sowie die damit zusammenhängende „besondere Einrichtung eines jeden Staats“ ${ }^{23}$

Gerhards ausführlicher Plan für eine preußische Bergschule enthielt auch eine Zusammenstellung der Kosten für den Erwerb von Laborinstrumenten und Modellen, aber keine Vorschläge zu Lehrergehältern, Stipendien oder den Ort der Schule. Dennoch kann er aufgrund der genauen Festlegungen über den Inhalt, die Dauer und die Abfolge des Unterrichts für die „Eleven“ als ein Plan für eine wirkliche Schule gewertet werden, der später noch in organisatorischer Hinsicht zu konkretisieren gewesen wäre. Dies geht auch aus der expliziten Rede von einer „vollständigen Berg-Schule“ hervor. ${ }^{24}$ Gerhards „Aufstellung der „zu diesem Etablissement nöthigen Kosten“, untermauert diese Interpretation, und sie zeigt vor allem auch, dass er nicht nur Vorlesungen, sondern auch Experimente und die Demonstration von Maschinenmodellen vorgesehen hatte. Schließlich fügte Gerhard noch eine Liste von vier „jungen Leuten“ hinzu, „die zu diesem Metier Lust“ hätten und als erste „Eleven“ in Frage kämen. ${ }^{25}$ 


\section{Eigenständige Bergschule oder Akademie der Wissenschaften?}

Bereits eine Woche nachdem Gerhard seinen Plan vorgelegt hatte, informierte ihn Minister von Hagen, der König habe seine Vorschläge zur „Einrichtung einer vollständigen Berg-Schule“ zwar im Prinzip gebilligt, jedoch vorerst zurück gestellt:

Ob nun zwar Höchstdieselben [die Königliche Majestät] die hierunter von ihm [Gerhard] gethane Vorschläge, als eine zur Anziehung tüchtiger Subjecte beym Bergbau dienende gute und nützliche Anstalt vollkommen approbiren, so muß doch die Ausführung derselben, wegen der dazu erforderlichen Kosten, bis zum Neuen nächst hervorbestehenden Etats Jahre ausgesezt bleiben. ${ }^{26}$

Der König war nicht bereit, die bescheidene Geldsumme von rund 410 Talern, die Gerhard für den Kauf der Laborausrüstung und Modelle berechnet hatte, sofort zur Verfügung zu stellen. Dies musste bei von Hagen Alarmglocken läuten lassen, der gleichzeitig auch mit Umstrukturierungen in der Verwaltung des Forst- und Bauwesens befasst war.

Der dirigierende Minister im Generaldirektorium von Hagen stand 1770 in der Tat vor einer Herkulesaufgabe. Er leitete das Bergwerks- und Hüttendepartment sowie das neue Forstdepartment, das erst im Januar 1770 gegründet worden war, und arbeitete darüber hinaus auch an einer Verwaltungsreform des zivilen Bauwesens. Gleichzeitig musste eine Ausbildungsreform der Beamten in diesen beiden Bereichen und im Bergwerks- und Hüttendepartment in die Wege geleitet werden. Die Organisation einer Bergschule bildete also nur einen kleinen Teil seiner Aufgaben, die zudem durch knappe Finanzen erschwert waren. Hinzu kam, dass er 1770 gesundheitlich so angeschlagen war, dass selbst eine „Brunnen-Cur“ im Sommer keine Besserung brachte. ${ }^{27}$ Von Hagen suchte daher nach pragmatischen Lösungswegen, und er fand diese im Meinungsaustausch mit seinem Ministerkollegen von Fürst. Seine Debatten mit von Fürst über die Ausbildungsreform der preußischen Beamten, denen im Folgenden nachgegangen wird, lassen neben bestimmten Grundlinien auch eine große Flexibilität erkennen. Von Hagen war überzeugt von der Nützlichkeit schulischen Wissens und von der Notwendigkeit, die nützlichen Wissenschaften staatlich zu fördern. Wie Gerhard, dessen Plan für eine Bergschule er unterstützte und daher unverändert an den König weiterreichte, strebte auch er eine wissenschaftliche Bildung aller preußischen Berg- und Hüttenbeamten an. Aber viele Fragen blieben zunächst noch offen und wurden erst allmählich und im Einvernehmen mit von Fürst geklärt.

Ernüchtert über die mangelnde Bereitschaft des Königs, schnell und längerfristig ausreichende finanzielle Mittel für einen wissenschaftlichen Unterricht seiner Berg- und Hüttenbeamten frei zu geben, lotete von Hagen umgehend alternative Möglichkeiten aus. Angesichts des engen finanziellen Spielraums war eine eigenständige Bergschule, die ausschließlich Beamte für das Berg- und Hüttenwesen unterrichtet hätte, fraglich geworden. Musste er 
unter diesen Bedingungen nicht von Anfang an über die Möglichkeit nachdenken, den wissenschaftlichen Unterricht für die zukünftigen Beamten der Berg- und Hüttenämter mit dem für die Beamten der Forst- und Bauverwaltung zusammenzulegen? Und gab es nicht leichter finanzierbare institutionelle Alternativen zu einer neuen Bergschule?

Bereits eine Woche nach dem Eintreffen der königlichen Antwort beauftragte von Hagen seinen Bergrat Gerhard, Erkundigungen über die Möglichkeiten und Finanzen der Akademie der Wissenschaften einzuholen. ${ }^{28}$ Die Königlich Preußische Akademie der Wissenschaften zu Berlin war in der Regierungszeit Friedrichs II. häufig mit Gutachtertätigkeiten für Erfindungen und technische Projekte befasst (Harnack 1900). Sie trug einen erheblichen Anteil an der Finanzierung des Collegium medico-chirurgicums, dessen anatomisches Theater im Marstall direkt neben den Räumen der Akademie lag (Lehmann 1936). Und mehrere Mitglieder der mathematischen und physikalischen Klasse der Akademie, darunter auch Gerhard, boten private oder öffentliche Vorlesungen in Berlin an. Unter diesen Bedingungen lag die Überlegung nahe, ob man die Akademie der Wissenschaften institutionell in die wissenschaftliche Ausbildung der preußischen Beamten einbinden könnte, zumal sie bereits über ein Laboratorium, eine Instrumentenkammer und einen botanischen Garten verfügte. Von Hagen beauftragte daher Gerhard, entsprechende Erkundigungen bei der Akademie einzuziehen. Am 14. Februar machte er seinem Ministerkollegen von Fürst den schriftlichen Vorschlag, die Akademie möge „practische Collegia“ lesen:

Außerdem wäre es gut und schicklich, daß das Institutum der Königlichen Academie der Wißenschafften, welche bekantermaßen gut dotirt ist, auch in denen practischen Theilen der desiderirten Wissenschaften mehr als geschiehet concurriren und practische Collegia lesen möge, zumalen in deren Fonds bey den Physicalischen und Mathematischen Classen folgende Posten jährlich ausgesetzt sind $[\ldots]^{29}$

Dem folgte eine Auflistung der Ausgaben der Akademie für ihr Laboratorium und ihre Instrumentenkammer sowie die Benennung von Akademiemitgliedern, die „nützliche Dienste“ leisten konnten. Johann Heinrich Lambert sollte Vorlesungen über Mathematik, Nicolas de Beguelin über Physik und Johann Gottlieb Gleditsch (1714-1786) über Botanik und Forstwissenschaft halten. Mit dem Verweis auf ihr Laboratorium und ihre Instrumentenkammer war die Akademie auch als Unterrichtsort ins Auge gefasst, obwohl der renommierte Chemiker Andreas Sigismund Marggraf (1709-1782), der damals Direktor des Laboratoriums und der physikalischen Klasse der Akademie war, nicht als Lehrer vorgesehen war.

Von Hagens Überlegungen zur Berliner Akademie der Wissenschaften als Ort der Ausbildung von Berg- und Hüttenbeamten erteilte der Geheime Etats- und Justizminister Carl Joseph Maximilian Freiherr von Fürst und Kupferberg (17171790) jedoch eine eindeutige Absage. ${ }^{30}$ Er verwies zunächst darauf, dass die 
Akademie der Wissenschaften keine Lehrinstitution sei, dass vielmehr „ihre Fundation und Einrichtung gantz unterschieden von solchen Academien, Universitaeten und Collegiis ist, welche aus Lehrern bestehen, die Lernenden Unterricht geben sollen“. ${ }^{31}$ Um jedes Missverständnis auszuschließen, fügte er hinzu:

Solche Gesellschaften wie die hiesige Academie der Wißenschafften [eine] ist, sind dazu bestimmt, das Reich der Wißenschafften und die menschliche Erkenntniß überhaupt, zu erweitern, solche auf einen höhern Grad der Vollkommenheit zu bringen, neue Entdeckungen zu machen, neues zu erfinden und durch Erfahrung zu bestätigen. ${ }^{32}$

Die Berliner Akademie habe große wissenschaftliche Erfolge zu verzeichnen, hieß es weiter, insbesondere in der Optik, Anatomie, Chemie und höheren Geometrie. Wenn man ein Mitglied der Akademie nun verpflichte, Unterricht zu erteilen, so werde ,ihm dadurch die Zeit entzogen werden, die er, in Rücksicht auf das gantze Reich der Gelehrsamkeit, viel nützlicher anwenden kann“ ${ }^{33}$

Obwohl diese Aufgabenbeschreibung der Berliner Akademie der Wissenschaften nicht ganz den Realitäten entsprach, und insbesondere ihre finanzielle und personelle Unterstützung des Unterrichts am Collegium medico-chirurgicum ignorierte, zog von Fürst hier eine klare Trennlinie zwischen der Akademie der Wissenschaften und einer Unterrichtsinstitution für Staatsbeamte. Vermutlich befürchtete er auch, dass die Akademie, die während des Siebenjährigen Krieges stark unterfinanziert war, einer neuen finanziellen Belastung Widerstand entgegen setzen würde, so wie sie dies bereits in früheren Jahren getan hatte als man ihr die Finanzierung des Anatomischen Theaters und des Collegium medico-chirurgicums übertragen hatte (Lehmann 1936: 12-13). Damit war der Vorschlag von Hagens jedenfalls vom Tisch, denn wenige Wochen danach versicherte dieser seinem Ministerkollegen er sei mit ihm ,in Absicht der Academie der Wißenschafften und dass derselben kein Unterricht anzumuthen sei, völlig einerlei Meinung." 34

Während sich von Hagen bemühte, eine eigenständige wissenschaftliche Ausbildung künftiger Berg- und Hüttenbeamter in der Residenzstadt Berlin zu ermöglichen, ergriff von Fürst, der auch Kurator der preußischen Universitäten war, Maßnahmen, die nützlichen Wissensbereiche an den preußischen Universitäten besser zu verankern. Diese Aktivitäten richteten sich ausschließlich auf die Anwärter für hohe Beamtenpositionen, für die eine Universitätsausbildung um die Mitte des 18. Jahrhunderts üblich war (Straubel 1998; Weber 1976). Insofern standen sie nicht notwendigerweise in Konkurrenz zu von Hagens Reformplänen, die sich ja auch auf mittlere und untere, technische Beamten erstreckten. Bereits im Dezember 1768 war die Universität Halle angewiesen worden, Vorlesungen über „Bergwercks-Wißenschafft" anzubieten..$^{35}$ Vom Januar 1770 an ergingen weitere Anordnungen an alle vier königlich preußischen Universitäten, von denen das Bergwerks- und Hüttendepartment in Berlin und die Oberbergämter 
in den Provinzen in Kenntnis gesetzt wurden. Das Berliner Bergwerks- und Hüttendepartment leistete dabei einerseits aktive Unterstützung. Andrerseits war von Hagen jedoch nicht sehr optimistisch gegenüber der Institution Universität als Ort der Lehre nützlicher Wissenschaften. Er begründete die Notwendigkeit einer neuen Lehrinstitution in Berlin auch damit, dass die „meisten Professores aber auf Universitaeten mehr speculativisch, als praktisch“ seien. ${ }^{36}$

\section{Erweiterte kameralistische Reformpläne}

Im Februar 1770 ging von Hagen nicht nur den institutionellen Alternativen $\mathrm{zu}$ einer Bergschule nach, sondern er stellte auch weitergehende inhaltliche Überlegungen über die Reform der Beamtenausbildung an. In einem Brief an von Fürst schlug er vor, einmal grundsätzlicher an die Frage der preußischen Beamtenausbildung heranzugehen. Es sei notwendig, so hieß es in diesem Brief, „daß man gemeinschafftlich von allen Seiten, endlich einmal ernsthaft darauf denke und würklich Hand anlege: wie bey der leyder so allgemeinen Unbrauchbarkeit eines großen Theils von Bedienten" und angesichts der Vielzahl der im königlichen Dienst stehenden „Idioten und Ignoranten“ zu verfahren sei. ${ }^{37}$ Zugleich aber brannte von Hagen darauf, trotz des finanziellen Vorbehalts des Königs, „wenigstens etwas ins Werck zu richten“ und schon in den kommenden Sommermonaten, ab dem ersten Juni, „öffentliche Collegia“ anzubieten, und zwar mit „zunächst mäßigen Honoraria“ für die Dozenten. Als Mittelweg zwischen einer umfassenden Ausbildungsreform für alle preußischen Beamten und dem pragmatischen Wunsch, wenigstens erste konkrete Schritte noch im Sommer 1770 in die Wege zu leiten, dachte von Hagen nun daran, künftige Berg- und Hüttenbeamten gemeinsam mit Beamten für das Bau- und Forstwesen unterrichten zu lassen.

Im Hinblick auf die Forstwissenschaften versprach von Hagen sich offenbar sehr unmittelbare praktische Auswirkungen von einer Ausbildungsreform. Die „schlechte Beschaffenheit der Forsten, und der bevorstehende Holz Mangel“, so äußerte er sich wenig später (am 17. Mai), hänge von der „großen Unwißenheit“ der „Forst-Bedienten“ ab, und hier sollten „öffentliche Collegia über ForstWißenschaffen“"von Gleditsch Abhilfe schaffen. ${ }^{38}$ Aber auch die „sehr neglirte“ Zivilbaukunst - von Wasserbau über Mühlenbau bis zu Bautätigkeiten im Bergbau und Hüttenwesen - bedürfe der Reformen. ${ }^{39}$ Daher ergriff von Hagen im April 1779 neben seiner Initiative zur Gründung eines eigenständigen Oberbaudepartments, eine weitere Maßnahme, die der „Unwissenheit und Unerfahrenheit der Bau-Bedienten“ entgegenwirken und „geschickte BauBediente" hervorbringen sollte. ${ }^{40}$ Auch hier stand es für von Hagen fest, dass dies nur unter Einbeziehung der Wissenschaften erreicht werden könnte. Das zu errichtende Ober-Baudepartment müsste daher ,jungen Leuten“ die Gelegenheit geben, „die hiezu nöthigen Wißenschafften, gründlich und practisch zu 
erlernen“. 41 Formulierungen wie „geschickte Bau-Bediente“ und „practisches Erlernen" verdeutlichen, dass von Hagen, ähnlich wie Gerhard, unter wissenschaftlichem Unterricht keineswegs nur Vorlesungen oder gar primär einen Unterricht von „Theorien“ im epistemologischen Sinn verstand; vielmehr bezog er auch technische Wissensbereiche und Know-how ein. ${ }^{42}$

Von Hagens Angebot vom 14. Februar, schon im Sommer 1770 in Berlin wissenschaftliche Vorlesungen für zukünftige Berg-, Hütten-, Forst- und Baubeamte anzubieten, stieß bei von Fürst auf offene Ohren. Aber es gab auch kleine Differenzen zwischen den beiden Ministern. Um einen „vollkommenen Cameralisten zu bilden“, so von Fürst in seinem Antwortschreiben an von Hagen vom 4. März, müsse ihm „eine ausgebreitete Kenntniß einer Menge von Wißenschafften und Künsten "vermittelt werden. ${ }^{43}$ Daher wolle er in nächster Zukunft eine „kurtze Encyclopedie aller Finantz- Oeconomie- PoliceyCommercien- Manufaktur- und dazu gehörigen Wißenschafften", erarbeiten lassen, „um mit einigen Blicken das weite Feld zu übersehen“. ${ }^{44}$ Anders als von Hagen, hatte von Fürst die gesamte Beamtenschaft im Auge und ging daher die Ausbildungsreform prinzipieller an. Erstens sollten schon „von jungen Jahren an“ nützliche Kenntnisse erworben werden, und der „Grund dazu schon auf Schulen“ gelegt werden. Hier begrüßte von Fürst ausdrücklich von Hagens vorangegangene Initiative, an der Berliner Realschule Physik, Mineralogie, Metallurgie und angewandte Mathematik unterrichten zu lassen. ${ }^{45}$ Zweitens waren die Universitäten einzubeziehen, und in dieser Hinsicht war von Fürst seit langem aktiv. Und drittens galt es zu bedenken, ob „noch andernwerts lehrreicher und zugleich praktischer Unterricht zu ertheilen sey “ ${ }^{46}$ Nur diese dritte und letzte Variante bezog sich auf von Hagens Plan für eine durch das Bergwerks- und Hüttendepartment organisierte Vorlesungsreihe in Berlin.

Von Fürst formulierte daher die Antwort auf von Hagens Vorschlag sorgfältig: Er sei mit seiner Absicht einverstanden, sich „vor der Hand auf diejenigen Wißenschafften einzuschränken, so die Bergwercke, das Bau-Wesen, und das Forst-Wesen unmittelbar angehen“ . ${ }^{47}$ Nur „vor der Hand“, also vorläufig, war er einverstanden, aber langfristig war die „Einschränkung“ nicht akzeptabel. Zudem präzisierte und korrigierte er von Hagens Vorschlag in zwei wichtigen Aspekten. Zum einen stellte sich die Frage des Verhältnisses öffentlicher Vorlesungen in Berlin zu den Universitäten. Hier stellte von Fürst klar, dass es prinzipiell zwei Möglichkeiten geben müsse. Die Berliner Vorlesungen konnten im Anschluss an ein Universitätsstudium oder unabhängig davon besucht werden. Letzteres war für Anwärter vorgesehen, die „nach ihrem Beruf gar nicht auf Universitaeten gehen", also für die mittleren und unteren Beamten mit technischen Aufgaben; ersteres für die hohen Beamten, die damals gewöhnlich an einer Universität studierten und denen auf diese Weise ,auch noch andere Gelegenheit zu verschaffen [sei], in allen oder einigen der oberwehnten Wißenschafften, Unterricht, besonders practischen zu erhalten" 48 


\section{Berlin als Ort für die Ausbildung von Berg- und Hüttenbeamten?}

Von Fürsts Insistieren auf praktischem Unterricht traf einen wunden Punkt in von Hagens Plänen. Es war kein Problem in Berlin, praktischen Unterricht in Baukunst zu erteilen, und auch die Forste in der Berliner Umgebung ermöglichten praktische Erfahrungen. Aber für das Berg- und Hüttenwesen war Vergleichbares ausgeschlossen - die preußischen Gruben und Hüttenwerke lagen weit entfernt von Berlin. Daher fügte von Fürst speziell für die Ausbildung der Berg- und Hüttenbeamten einen wichtigen Vorbehalt hinzu: Berlin könne für den Unterricht nur eine vorläufige Lösung der Berg- und Hüttenbeamten sein. Wörtlich hieß es dazu, Berlin sei ein geeigneter Standort nur

solange nicht wegen der Bergwercks-Wißenschafften, nach dem Exempel des vor einigen Jahren zu Freyberg in Sachsen gemachten Instituti [...] zu theoretischen und practischen Unterweisungen, bey einem der Königl. Berg-Aemter selbst, Anstalt gemacht werden kan. ${ }^{49}$

Von Fürst ließ also keinen Zweifel daran aufkommen, dass er eine Verbindung von Vorlesungen und praktischer Lehre in der Ausbildung der Berg- und Hüttenbeamten für notwendig halte, und er untermauerte dies noch durch den Hinweis auf die Bergakademie in Freiberg. Ähnliche Vorbehalte gegen eine Bergschule in der Residenzstadt Berlin teilte später auch der für das Bergund Hüttenwesen zuständige preußische Minister Friedrich Anton von Heynitz (ab 1777). Dieser hatte an der Gründung der Bergakademie in Freiberg mitgewirkt, und er hatte ähnliche Debatten auch in Paris, im Vorfeld der Gründung der École des Mines erlebt (Weber 1976: 173-177).

\section{Theorie und Praxis in Freiberg: Gerhards Bericht}

Von Fürsts Hinweis auf die Freiberger Bergschule hatte sofortige Konsequenzen. Unmittelbar danach wurde Gerhard nach Freiberg geschickt, um detaillierte Informationen einzuholen. Nach Berlin zurückgekehrt, legte er am 24. März seinen Bericht vor. ${ }^{50}$ Dieser Bericht veranlasste von Hagen, Gerhards Plan für eine Bergschule aufzugeben. Denn dieser führte nicht nur deutlich vor Augen, dass es in Freiberg ungleich bessere finanzielle Bedingungen gab als in Berlin, es war dort vor allem auch möglich, den schulischen Unterricht mit der praktischen Lehre in Gruben und Hüttenwerken zu verbinden. Freiberg war eine Bergstadt, wo Gruben und Hüttenwerke in wenigen Minuten erreichbar waren und in der seit Jahrhunderten nahezu das gesamte wirtschaftliche, kulturelle und soziale Leben auf den Bergbau abgestimmt war. Vergleichbares hatte Berlin nicht zu bieten.

Gerhard berichtete, es gäbe in Freiberg zwei Professoren, nämlich den Bergrat, Mineralogen und Chemiker Christian E. Gellert, der die Metallurgie und Hüttenlehre lese, und Johann F. W. Charpentier, der Mechanik, 
praktische Geometrie und Zeichenkunst lehre. Charpentier habe für seinen Unterricht auch Modelle, Zeichnung und Risse angeschafft und erhalte für deren Unterhaltung jährlich 150 Taler. Auch Gellerts Laboratorium wurde erwähnt, insbesondere die Tatsache, dass er für seine Experimente jährlich 200 Reichstaler erhalte. Weiterhin berichtete Gerhard, es studierten in Freiberg zurzeit sechs junge „Berg Cadetten“, und zwar auf "landesherrliche Kosten“, da sie Landeskinder waren. Nach einem "Cursum" von zwei Jahren gingen sie auf Reisen, und auch dafür erhielten sie finanzielle Unterstützung aus der Bergkasse. In nur wenigen Sätzen hatte Gerhard somit insgesamt viermal das Problem der Finanzierung angesprochen, das ihm auch wegen eines Laboratoriums am Herzen lag.

Dann kam das Verhältnis von Theorie und Praxis zur Sprache, über das sich Gerhard in seinem Plan für die Berliner Bergschule eindeutig festgelegt hatte, denn er hatte gefordert, dass alle Schüler zuerst eine einheitliche zweieinhalbjährige schulische Ausbildung durchlaufen sollten, bevor sie praktische Instruktionen im Gruben- oder Hüttenwesen erhielten. Da jedoch in Freiberg die Vorlesungen mit praktischem Gruben- und Hüttenunterricht verbunden waren, war es nur konsequent, dass Gerhards Bericht in diesem Punkt kritisch ausfiel. Theoretischen oder schulischen Unterricht über den Grubenbau, so wie er es in seinem eigenen Plan vorgesehen hatte, gab es in Freiberg nicht. Stattdessen wurden diese Kenntnisse „ex Usu und bey der täglichen Befahrung der Gruben erlernt". Ergänzend fügte er hinzu, dass

diese Einrichtung noch sehr incomplett sey, da in dem Waßer Bau, dem Forst Wesen und dem Bergbau selbst kein Unterricht gegeben, sondern der letztere bloß practisch erlernet wird, welches ein absolutes Hindernüß abgiebt, dass dieses wichtige Metier $(\mathrm{zu})$ in eine ordentliche und systematische Verfassung komme. ${ }^{51}$

Schließlich fiel auch die praktische Herangehensweise an die Köhlerei der Kritik Gerhards anheim.

Was Gerhard erst gar nicht erst explizit erwähnte war die Tatsache, dass in Freiberg außer den Professoren Gellert und Charpentier auch Praktiker unterrichteten, nämlich ein Probierer (oder „Bergwardein") namens Johann A. Klotzsch, ein Markscheider namens Carl E. Richter sowie ein Bergmeister namens Christian H. Lommer, der die Mineraliensammlung der Bergakademie betreute und vorführte. Die Probier- und Markscheidekunst wurden in Freiberg schon lange vor dem 18. Jahrhundert nicht nur praktisch-handwerklich durch Beobachten, Nachahmen und Einüben erlernt. Freiberg war vielmehr seit dem 16. Jahrhundert ein bedeutender Ort für die Entstehung und Verbreitung bergbaulicher Schriften. Die handwerkliche Lehre der Probier- und Markscheidekunst integrierte vermutlich schon seit dieser Zeit mathematische Übungen und Texte (Baumgärtel 1965, Herrmann 1953). Die technologischen Texte über die Markscheide- und Probierkunst, die im 17. Jahrhundert von Freiberger Bergbeamten geschrieben wurden, ergänzten die Vermittlung lokalen und teilweise geheimen, mündlichen Wissens und Know-hows. Ab 1702 vergab 
das Freiberger Bergamt zudem Stipendien für das Erlernen der „Probier- und Markscheidekunst" und, wie es hieß, der "Bergk- und Schmelz-Wissenschafft" (ebd.: 117). Freiberg galt aufgrund dieser Lehrtradition schon im frühen 18. Jahrhundert als Zentrum avancierter Bergbaukenntnisse (ebd., Wagenbreth 1994). Um die Mitte des 18. Jahrhunderts wurde es insbesondere durch den Privatunterricht und das Laboratorium des Bergrats und Mineralogen Johann Friedrich Henckel weit über die Landesgrenzen hinaus bekannt (Herrmann 1962).

Somit war in Freiberg für die mittleren und unteren Beamtenränge schon früh eine Mischform zwischen handwerklicher und schulischer Lehre eingeführt worden, die mit der Gründung der Freiberger Bergakademie im Jahr 1765 nicht abrupt beendet wurde. Vielmehr wurde hier auch eine gewisse Kontinuität der handwerklich-praktischen Anteile des Unterrichts bewahrt, was nicht zuletzt dadurch gewährleistet wurde, dass die bis dahin mit der Lehre beauftragten Probierer und Markscheider zu Lehrern der Bergakademie ernannt wurden. Hinzu kam das tägliche Einfahren der Bergkadetten in die Gruben, das Gerhard direkt bezeugte, beziehungsweise das Erlernen von Hüttentechniken direkt in der Hütte.

\section{Von Hagens Taktik}

Nach Gerhards Bericht muss für von Hagen sofort festgestanden haben, dass die Freiberger Bergakademie beim König nicht als Vorbild für die Berliner Bergschule angeführt werden konnte. Die Bedingungen waren zu unterschiedlich. Am nächsten Tag, dem 25. März, suchte er eine vier Jahre alte Anordnung Friedrichs II. an die Kurmärkische Kammer heraus, die eine herbe Kritik des Königs am Baudepartment der Behörde enthielt und strenge Maßnahmen ankündigte. ${ }^{52}$ In einer Randnotiz hielt er nun fest:

Ich glaube, daß, um bei des Königs Maj. desto ehender die Genehmigung des Vorhabens in Absicht der Beförderung der practischen Wissenschaften zu effectuieren und die Etablissements nicht zu vervielfältigen, man das generale $\overline{\text { Baudepartment mit unsern schon präparirten Etablissements beim Bergwerks- }}$ department combiniren und ersteres in verschiedene Klassen eintheilen [soll].

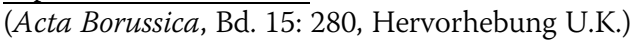

Von Hagen wusste also seit langem, dass der König besonderes Interesse an der Verbesserung der Zivilbaukunst hatte. Nun stand sein Entschluss fest, der Vermerk in der Randnotiz reifte in den nachfolgenden zwei Wochen zu einer ausgeklügelten Taktik heran. ${ }^{53}$

Am 7. April 1770 legte von Hagen daher ein Memorandum vor, das sich in erster Linie mit der Frage befasste „wie man das in denen sämtlichen Provintzen so sehr neglirte Bau-Wesen, auf einen bessern Fuß zu setzen " habe. ${ }^{54}$ Es enthielt Vorschläge für die Ausbildung der Bau- und Forstbeamten, in deren Kompetenzbereich das wichtigste Baumaterial - Holz - fiel, schloss jedoch, listig verpackt, auch die Ausbildung der Bergbeamten ein. Denn in dem Memorandum hieß es: 
Damit außerdem junge Leute, die sich auf das Bau-Wesen, die Forst-Sachen, und den Berg-Bau legen wollen, eine bequeme Gelegenheit haben mögen, sich zum künftigen Dienst gründlicher zu qualificiren, so würde es sehr gut seyn, wenn alhier [in Berlin] Gelegenheit verschaffet würde, die hiezu nötigen Wissenschaften, gründlich und practisch zu erlernen. ${ }^{55}$

Dem folgte eine gemeinsam mit von Fürst erarbeitete Liste der Lehrer (siehe unten), die diesen Unterricht ab 1. Juni 1770 abhalten sollten.

Der König genehmigte von Hagens Plan umgehend und zeichnete am 17. April eine ausführliche „Instruction für das angeordnete Ober-Baudepartment“ ab. $^{56}$ Von Hagens kleine List blieb unbemerkt, denn die vom König abgezeichnete Instruktion enthielt eine nicht unwesentliche Variante der ursprünglichen Textstelle. Während dort von Unterricht für ,junge Leute, die sich auf das Bau-Wesen, die Forst-Sachen, und den Berg-Bau legen wollen“ die Rede war, hieß es nun: „um jungen Leuten, die sich auf die Baukunst appliciren wollen und dazu Lust haben, die Gelegenheit zu dem nöthigen gründlichen Unterricht zu verschaffen", sollten vom ersten Juni an öffentliche Vorlesungen eingerichtet werden (Acta Borussica, Bd. 15: 291, Hervorhebung U.K.). Der Vorfall ist instruktiv für die Taktik, die ein reformfreudiger Minister wie von Hagen einschlagen musste, um Gestaltungsspielraum und ein machtpolitisches Gegengewicht zum König und seinem Kabinett in Potsdam zu erreichen. ${ }^{57}$ In diesem Fall war die Taktik von Erfolg gekrönt, denn die geplante Vorlesungsreihe wurde von höchster Stelle genehmigt. Zwei Tage später, am 19. April, konnte von Hagen daher von Fürst berichten, seine Vorschläge für die „Erweiterung der Finantz- und Cameral-Wißenschafften“ und „die Ausbreitung der Wißenschafften in Absicht der Berg-Werke[,] des Bau- und Forstwesens" seien „mit wahrem Vergnügen“ angenommen worden. ${ }^{58}$

Damit aber gehörte der Plan Gerhards für eine Bergschule, die nur Bergund Hüttenbeamte ausbildete, endgültig der Vergangenheit an. An die Stelle eines systematischen Unterrichts im Rahmen einer Schule trat nun ein unverbindliches Angebot öffentlicher Vorlesungen, die gelegentlich durch andere Angebote, wie Zeichenunterricht und Laborbesuche, ergänzt wurden.

\section{Lehrer für ein Douceur?}

Im April hatten die beiden Minister somit vereinbart, in Berlin eine staatlich unterstützte Vorlesungsreihe für künftige Berg- und Hüttenbeamte sowie für Forst- und Baubeamte einzurichten. Die Physikvorlesung sollte der am Collegium medico-chirurgicum lehrende Professor Johann Gottlieb Walter (1734-1818) halten, zumal dieser bereits die „sonst seltenen und kostbaren Instrumente zur Experimental-Physic" besaß; hier konnte also Geld gespart werden. ${ }^{59}$ Für die Chemie war der Apotheker Valentin Rose (1736-1771) vorgesehen, der „bey dem grossen Chimico H[errn] Marggraff ausgebildet“ 
worden war. ${ }^{60}$ Die Mineralogie und Metallurgie sollte Gerhard unterrichten, und zwar im Winter, wenn er nicht auf Inspektionsreisen war, und die Vorlesungen in Forstwirtschaft sollte Johann Gottlieb Gleditsch halten, der wie Walter am Collegium medico-chirurgicum unterrichtete und sich offenbar schon bereit erklärt hatte, für „ein billiges Honorarium“ zu lesen. ${ }^{61}$ Für die Mathematikvorlesung hatte von Hagen eine besondere Regelung vor, denn hier sollte zweigleisig verfahren werden. Die künftigen Berg- und Hüttenbeamten sollte der Oberkonsistorialrat und Direktor der Berliner Realschule Johann Esaias Silberschlag (1721-1791) unterrichten, während für die Interessenten der Baukunst Friedrich Adolf M. Castillon (Castillion der Jüngere, 1747-1814) eingeplant war. ${ }^{62}$

Für diese Auswahl an Lehrenden dürfte neben ihren Kenntnissen auch ihr sozialer Status ausschlaggebend gewesen sein. Es galt abzuwägen, wer der neuen Vorlesungsreihe einerseits ausreichendes Renommee verschaffte, andrerseits aber durch ein "Douceur" von jährlich 100 Talern nicht düpiert war. $^{63}$ So hätte beispielsweise Andreas Sigismund Marggraf, Direktor des Laboratoriums und der physikalischen Klasse der Akademie der Wissenschaften, als berühmtester Chemiker Preußens der Vorlesungsreihe zweifellos Glanz verliehen, aber er kam wegen seiner Position als Akademiedirektor und vielleicht auch aus Altersgründen (er war 61 Jahre alt) nicht in Betracht. Der Apotheker Valentin Rose dagegen war erst 34 Jahre alt. Er hatte bei Marggraf gelernt, war der Besitzer einer angesehenen Apotheke und darüber hinaus auch Assessor im Obercollegicum medicum, der obersten Medizinalbehörde Preußens. Vor allem aber besaß Rose als Apotheker auch ein eigenes Laboratorium, so dass er ohne größere Zusatzkosten Experimente vorführen konnte. Ähnliche Erwägungen dürften umgekehrt auch für die Lehrer eine Rolle gespielt haben. Das Honorar war, trotz der zu erwartenden zusätzlichen Hörergelder, nicht gerade verlockend. Dem stand aber die ehrenvolle Anfrage des Bergwerks- und Hüttendepartments gegenüber. Wie also reagierten die Befragten auf von Hagens Angebot?

Gleditsch bedankte sich umgehend für das Angebot und versicherte, er werde „die Gründe der Oeconomisch Praktischen Botanic, als eines Grund Studii beym Cameralwesen [...] einem jeden sehr gerne begreiflich [...] machen, welcher sich zu solchen Königlichen Diensten gehörig zu qualificiren Lust hat" ${ }^{64}$ Auch Gerhard akzeptierte sofort. ${ }^{65}$ Ebenso bedankte sich Walter im August für die ihm ,gnädigst anvertraute Stelle eines Lehrers der Experimental Physic." ${ }^{\text {"66 }}$ Oberkonsistorialrat Silberschlag dagegen beantwortete das „höchstschätzbare Merkmahl eines huldreichen Zutrauens" zwar mit ausgesuchter Höflichkeit, sagte jedoch aufgrund der Verpflichtungen in seinem Kirchenamt und als Direktor der Berliner Realschule ab. ${ }^{67}$ Dies führte schließlich dazu, dass die Mathematikvorlesungen gänzlich von Castillon (dem Jüngeren) übernommen wurden, der ursprünglich nur die Interessenten der Baukunst unterrichten sollte. 
Die Reaktion Castillons auf das Angebot des Bergwerks- und Hüttendepartments ist mindestens aus zwei Gründen interessant. Erstens war er der einzige, der zusätzlich Informationsfragen stellte und deutlich zu erkennen gab, dass das Angebot nicht unbedingt als ein Aufstieg in der sozialen Hierarchie zu werten war. Wie häufig solle er unterrichten? Könnte er das Hörerhonorar und die Zahl seiner Schüler selbst festlegen? Welchem Minister unterstünde er? Und war mit dem Unterricht auch der Professorentitel verbunden? Zweitens enthielt die Antwort von Hagens auf die letzte Frage Castillons eine wichtige Information darüber, wie der Minister selbst einen Monat vor Beginn der Vorlesungen, die neue Institution verstand. ${ }^{68}$ Von Hagen beantwortete am 24. September 1770 die Frage Castillons nach dem Professorentitel abschlägig und begründete dies damit, dass

die gegenwärtig veranlaßte Docirung verschiedener Wißenschafften, eben kein neues Institutum, sondern nur bloß der Zweck dabey ist, dass jungen Leuten in solchen nützlichen Wissenschaften, die bishero noch zu sehr verabsäumet worden, Unterricht ertheilet und dem Staate dadurch brauchbare Subjecta zugezogen werden $[\ldots . .]^{69}$

Ein Professorentitel wurde also deshalb nicht vergeben, da es sich nicht um eine wirkliche Schule handelte. ${ }^{70}$

In einem Brief vom 17. September 1770 reagierte auch Valentin Rose eher verhalten auf das Unterrichtsangebot. Er verwies auf seinen schlechten Gesundheitszustand, seine Mitarbeit bei Zeitschriften und im Obercollegium medicum und insbesondere seine Auslastung als Apotheker: „Ew. Hochedelgebohren werden leicht einsehen, wie ich allemahl die pflichtmäßige Besorgung meiner Apotheke zum Haupt-Augenmerk behalten muß und daß deren Ordnungs mäßiger Betrieb, eine beständige Aufmerksamkeit erfordert." Dennoch erklärte er sich bereit, den „Auftrag“ zu übernehmen, wenn die Bereitschaft bestand, nicht nur eine Vorlesung, sondern auch einen experimentellen Kurs in Chemie zu finanzieren. ${ }^{71}$ Roses Absage im Januar 1771 war auch der mangelnden Bereitschaft geschuldet, ihm hier entgegenzukommen (siehe unten).

\section{Unkoordinierte Vorlesungen statt Bergschule}

Das Bergwerks- und Hüttendepartment legte den Beginn seiner Vorlesungen auf den 15. Oktober 1770 fest und machte dies der Öffentlichkeit durch Zeitungsannoncen bekannt. ${ }^{72}$ Keiner der involvierten Akteure verstand die neue Vorlesungsreihe in Berlin als eine „Schule“ oder gar als eine neue „bergtechnische Hochschule“, wie dies insbesondere von Paul Krusch behauptet worden ist (Krusch 1904: IX f.). Erstens wandte sich das Vorlesungsangbot auch mit gleichem Nachdruck an zukünftige Beamte des Bau- und Forstwesens, und zweitens war im Herbst 1770 fast durchweg von „Vorlesungen“ die Rede. Dies 
war auch deshalb angemessen, weil weder ein Unterrichtsgebäude noch einen Stundenplan (wie etwa am Berliner Collegium medico-chirurgicum) oder auch nur eine zeitliche Koordination der einzelnen Vorlesungen vorhanden waren, denn Ort und Zeit und der Vorlesungen waren jedem Dozenten individuell überlassen. Ein Stipendium war nicht vorgesehen, und auch dies war ein Unterschied zu existierenden Schulen wie der Bergakademie Freiberg und dem Berliner Collegium medico-chirurgicum. Schließlich gab es auch keine formelle Immatrikulation und keine Prüfungen wie am Collegium medico-chirurgicum. Das Bergwerks- und Hüttendepartment kam lediglich für das bescheidene Lehrerhonorar von jährlich 100 Talern auf sowie für einige Instrumente, die Gerhard für seine Vorlesungen benötigte sowie für Bücher für die Bibliothek des Bergwerks- und Hüttendepartements. ${ }^{73}$ Erst im November 1774 wurde ein Laboratorium im Hof von Gerhards Wohnung in der Allee Unter den Linden eingerichtet, das sowohl für Gerhards Forschung als auch für seinen Unterricht zur Verfügung stand. ${ }^{74}$ In den nachfolgenden Jahren wurden Modelle, Landkarten, Grubenrisse, physikalische und mathematische Instrumente und eine Mineraliensammlung erworben, so dass Nicolai 1786 von einem „Kabinett“ des Bergwerks- und Hüttendepartments sprechen konnte (Nicolai 1786: 804-806). Wie das Laboratorium, so hatte auch dieses Kabinett verschiedene, nicht nur auf den Unterricht bezogene Funktionen.

Im Vergleich zu den in Berlin seit langem angebotenen Privatvorlesungen wurden 1770 jedoch zwei nicht ganz unbedeutende Neuerungen eingeführt. Erstens konnte das jährliche Honorar trotz seiner Bescheidenheit als Instrument zur Anbindung der Dozenten an die Behörden eingesetzt werden. Denn im Gegenzug waren die Dozenten verpflichtet, regelmäßig über ihre Vorlesungen Bericht $\mathrm{zu}$ erstatten und eine Liste der Hörer einzureichen, die Angaben über deren Herkunft und berufliche Absichten enthielten. Die im Sommer 1770 eingerichteten öffentlichen Vorlesungen des Bergwerks- und Hüttendepartments wurden nicht zuletzt durch diese Verpflichtungen der Dozenten im Laufe der Zeit zu einem tatsächlichen Rekrutierungsfeld für den preußischen Verwaltungs- und Beamtenapparat.

Zweitens nahm das Bergwerks- und Hüttendepartment auch Einfluss auf die Lehrinhalte, insbesondere wenn der Verdacht aufkam, dass eine Vorlesung nicht wirklich nützlich war. Ein instruktives Beispiel dafür liefern die Umstände der Ernennung des Berliner Arztes Ernst Gottfried Kurella (1725-1799) als Dozent für Chemie. Nachdem Rose abgesagt hatte wandte sich Kurella umgehend (noch im Januar 1771) an das Bergwerks- und Hüttendepartment mit dem Angebot, er könne neben seiner ärztlichen Praxis noch Zeit für eine Chemievorlesung aufbringen. ${ }^{75}$ Nur zögernd erteilte das Bergwerks- und Hüttendepartment Kurella im März 1771 eine Zusage, denn offenbar gab es Bedenken, dass der Arzt vorwiegend pharmazeutische Chemie unterrichten werde. Diese Zusage war daher verbunden mit der Anweisung, er müsse die "Oekonomie und das Cameralwesen" angemessen berücksichtigen und 
„besonders über die Gährungen, Färbereien, Salpeter-Machen, künstliche Düngungen der Aecker pp. Versuche anstelle[n]“, jedoch „alles was in die Chymiam pharmaceuticam einschlägt weglassen " ${ }^{76}$

Vor allem die Vorlesungen Gerhards und Gleditschs waren von Anfang an gut besucht. Gerhard hatte im ersten Jahr 22 und Gleditsch 17 Hörer, darunter viele angehende Mediziner und Apotheker, aber auch mehrere, bereits im Staatsdienst stehende Beamte. Beide Dozenten reichten auch die erwarteten Hörerlisten ein, wobei sich allerdings zeigte, dass im ersten Jahr nur ein einziger ihrer jungen Hörer Bergbeamter werden wollte. ${ }^{77}$ Die Mathematikvorlesung Castillons wurde dagegen nach einem halben Jahr wieder eingestellt. Nachdem ihn das Bergwerks- und Hüttendepartement im Mai 1771 an die einzureichende Hörerliste erinnerte, bat er kurz darauf um seine „Demission“ “ ${ }^{78}$ Dem war eine Intrige des jungen Referendars Friedrich Holsche (1743-1783) vorangegangen, der kurz danach zum Nachfolger Castillons und zum Oberbaurat ernannt wurde (Acta Borussica Bd. XV: 280, Bd. XVI.2.: 995). Im Januar 1771 hatte sich Holsche direkt an den König mit der Bitte gewandt, Vorlesungen in praktischer Geometrie und Zivilbaukunst halten zu dürfen; ${ }^{79}$ ein Monat später wurde ihm diese Erlaubnis erteilt. ${ }^{80}$ Damit gab sich Holsche jedoch nicht zufrieden. Im Mai erklärte er dem Generaldirektorium, er könne sehr viel nützlichere, auch durch Instrumente und Modelle gestützte Vorlesungen halten, wenn dies nicht „aus Mangel der Subsistence“ verhindert würde. Nachdem er dann explizit die neue Vorlesungsreihe des Bergwerks- und Hüttendepartments in Spiel gebracht hatte sowie die Tatsache, dass dafür „besondere Gehälter“ bezahlt würden, bezichtigte er Castillon, nur die „mathesia puram“ zu lesen; daraus aber, so Holsche, könne „weder der König noch das Land jemals Nuzzen ziehen" ${ }^{81}$

Ähnlich erging es der Chemievorlesung, die Valentin Rose erst gar nicht antrat. Nachdem Gerhard im November 1770 auf einer Konferenz des Bergwerks- und Hüttendepartments beauftragt worden war, Erkundigungen über die Vorlesungen Roses einzuholen, erhielt er von diesem eine ,ziemlich sonderbare Antwort". ${ }^{2}$ Rose antwortete, er habe „noch gar nicht angefangen“, weil die Honorarfrage noch offen sei. Rose ging es aber nicht nur um Geld, es handelte sich hier auch um einen inhaltlichen Konflikt über die Frage, was nützliches Wissen sei und wie es unterrichtet werden könne. ${ }^{83}$

\section{Reformvorschläge für den praktischen Unterricht}

Der Apotheker und Chemiker Valentin Rose hatte sehr genaue Vorstellungen, welche Bereiche chemischen Wissens und welche Art von Chemieunterricht als nützlich gelten konnten. So hatte er bereits bei der ersten Anfrage des Bergwerks- und Hüttendepartments geltend gemacht, dass die Schüler „nicht mühsam durch den gantzen Unterricht der Theorie geleitet, sondern 
hauptsächlich mit der practischen Chemie bekandt gemacht" werden sollten. ${ }^{84}$ Der Terminus "practische Chemie“ hatte hier eine Doppelbedeutung. Zum einen bezog er sich auf chemische Tätigkeiten außerhalb der Schule, beispielsweise im Laboratorium des Apothekers, und zum anderen meinte er experimentellen Laborunterricht in der Schule, der teilweise auch gewerblichchemische Operationen imitierte. Daher sollte der "Process der Operationen“ im Vordergrund des experimentellen Unterrichts stehen.

Practischer Unterricht bedeutete jedoch, dass Rose in seinem kleinen Apothekerlaboratorium nicht mehr als sechs Schüler unterrichten konnte, „weil sonst der Process der Operationen nicht einem Jeden genau genug demonstrirt werden könnte". Darüber hinaus erklärte Rose, dass ein solcher „Cursus chymischer Operationen“ einiges kosten würde: „Auf das mindeste gerechnet würde ein solcher Cursus chymischer Operationen, an Utensilien, dazu gehörigen Droguerien, Kohle und sonstigen Materialien 300 Th[aler] baar, allein zu stehen kommen". ${ }^{55}$ Von Hagens Antwort ließ nicht lange auf sich warten. Rose erhielt ein Lob - er war „wegen Seiner bekandten Geschicklichkeit in dieser Wißenschafft vorzüglich recommandiret worden" aber zusätzliches Geld für den Laborunterricht war nicht zu erwarten; stattdessen sollte sich Rose ein „billiges Honorarium“ von seinen „Auditoren“ holen. ${ }^{86}$ Dies war jedoch angesichts der geringen Schülerzahl für einen experimentellen Kurs keine befriedigende Lösung.

In seiner Antwort auf die Nachfrage Gerhards vom November 1770 äußerte sich Rose daher noch präziser über die Notwendigkeit experimentellen Unterrichts. Die Teilnehmer seines Kurses sollten „selbst Gelegenheit bekommen mit Hand anzulegen“. Es ging jetzt also nicht mehr allein um Demonstrationsexperimente, sondern um einen experimentellen Kurs unter aktiver Beteilung der Teilnehmer, dessen Dauer Rose auf sechs bis neun Monate festgesetzte, in Abhängigkeit von seinem Gesundheitszustand. ${ }^{87} \mathrm{Zu}$ den Voraussetzungen eines solchen Unterrichts gehörten natürlich auch die finanziellen Mittel, und auch dazu äußerte sich Rose nun mit unmissverständlicher Klarheit: „Ich habe darinne [im Brief an von Hagen vom September] meine Meinung offenherzig geschrieben, wie und auf welchem Fuß ich willens sei die Chimie vorzutragen, und dabei werde ich auch bleyben, es müßte den sein, daß sich andere fänden, die um des liben brodts willen wohlfeiler arbeiteten“. ${ }^{88}$ Rose gab hier klar zu verstehen, er sei nicht genötigt, für jedes beliebige Honorar zu arbeiten. Dem fügte er die ironische Bemerkung über die im doppelten Sinne „kostbare Chemie“ hinzu, die Gerhard durchaus persönlich nehmen konnte.

Da Ew. Wohlgeb[oren] selbst die Chymie gelehrt. [...], so wird es ihnen am besten bekannt sein, wie eine kostbahre Sache die Chymie ist, auch daß eine große Anzahl Zuhörer niemahle den Nutzen davon haben werden [,] den wenige Zuhörer haben, wenn sie selbst Gelegenheit bekommen mit Hand anzulegen. Freylich kostet es ihnen dann mehr, aber der Nutzen ist auch für Sie evidenter. ${ }^{89}$ 
Es wundert nicht, dass Gerhard diese Antwort „sonderbar" fand, denn dies war in der Tat nicht der Ton, den man in preußischen Behörden gewohnt war. Als Bergrat hatte er die Gesichtspunkte des Bergwerks- und Hüttendepartments zu vertreten, obwohl ihm als experimentierendem Naturforscher, der selbst die Einrichtung eines Laboratoriums für die zukünftigen Berg- und Hüttenbeamten vorgeschlagen hatte, die Argumente Roses einleuchten mussten. Der Disput wurde schließlich im Januar 1771 mit einem Brief Roses beendet, in dem er aus Krankheitsgründen absagte. ${ }^{90}$

Roses entschiedenes Eintreten für einen experimentellen Kurs der Chemie anstelle bloßer Vorlesungen war kein individueller Alleingang. Es wird vielmehr verständlich, wenn man die in Berlin bereits bestehende Tradition experimentell-chemischen Unterrichts am Collegium medico-chirurgicum berücksichtigt, das 1724 für Chirurgen (hauptsächlich Militärchirurgen) und daneben auch für Mediziner und Apotheker gegründet worden war. An diesem neuartigen Lehrinstitut gab es zwei Professuren für Chemie, davon eine für die praktische Chemie („chymia practica“), die im Laboratorium der Hofapotheke gelehrt wurde. Der Apotheker und renommierte Chemiker Caspar Neumann (1683-1737) hatte dort Maßstäbe für den experimentellen Chemieunterricht gesetzt. Auch Johann Heinrich Pott (1692-1777), der nach wie vor die Professur für "rationale pharmazeutische Chemie" an diesem Kollegium innehatte, war ein geschickter Experimentator, der auch nützliche experimentelle Projekte, wie das zur Herstellung von Porzellan, unternahm. Marggraf, der um 1770 unbestritten führende preußische Chemiker, hatte den größten Teil seiner Apothekerlehre bei Neumann in der Hofapotheke verbracht, und auch er war ein theoretischer Skeptiker, der stets die Notwendigkeit des experimentellen Vorgehens in der Chemie betonte. Schließlich hatte auch Valentin Rose selbst am Collegium medico-chirurgicum studiert (Lyncker 1934: 141).

Wie Rose, so setzte sich auch Gerhard für experimentellen Unterricht ein. Dies war, ebenso wie die Anwendung von Modellen, bereits in seinem Plan für eine Bergschule vom Januar 1770 vorgesehen, und schon lange vor der Errichtung eines Laboratoriums im Spätherbst 1774 muss Gerhard Experimente vorgeführt haben, für die er einen Probier- und Windofen und eine Probierwaage anschaffte. ${ }^{91}$ Im November 1774, unmittelbar nach der Einrichtung eines Laboratoriums, konnte Gerhard dann berichten, er halte Dienstags und Mittwochs jeweils eine Stunde Vorlesungen, in denen „die Mineralischen Cörper selbst denen Auditores vorgezeigt" werden. Da die Schüler das Probieren der Mineralien jedoch auch durch eigene Versuche erlernen sollten, habe er „zu denen Versuchen aber den Sonnabend Nachmittag von 2 Uhr an bestimmet". 92

Auch Gleditsch beschränkte seinen Unterricht über Forstwissenschaften nicht auf die traditionellen Vorlesungen, sondern unternahm mit seinen Schülern Exkursionen in den Berliner Forst. Auf diesen Exkursionen, die er 
nach eigenen Angaben auch für Unterricht über „Forstwirtschaft“ nutzte, ging er auch auf aktuelle Probleme der forstwirtschaftlichen Praxis ein. Zu diesem Zweck bat er das Bergwerks- und Hüttendepartment, ihm amtliche Unterlagen über Feuer- und Sturmschäden, Dürreperioden, Schädlingsbefall usw. zur Verfügung zu stellen. Eine entsprechende Anweisung für die Aushändigung der Akten erging umgehend an Registrator Schulz, so dass es hier zu einem direkten Wissenstransfer zwischen der Behörde und einem unterrichtenden Wissenschaftler kam. ${ }^{93}$

\section{Nützlichliche Wissenschaften im absolutistisch-merkantilistischen Staat}

Nach dem Ende des Siebenjährigen Krieges unternahmen die preußischen Behörden eine Reihe von Maßnahmen für die Verbesserung der Gewerbepolitik und Behördenstruktur. Die nach dem Krieg einsetzende Wirtschaftskrise, die finanziellen, technischen und logistischen Bedürfnisse der preußischen Armee und die zunehmende wirtschaftliche Konkurrenz infolge der rapiden Industrialisierung Englands und der staatlichen Wirtschaftsinitiativen in anderen Residenzstädten wie Dresden, Wien und Paris führten den Handlungsbedarf nur allzu deutlich vor Augen. Die seit Jahrzehnten in Preußen praktizierte kameralistische Wirtschaftspolitik war, wie in anderen absolutistisch-merkantilistischen Staaten, mit der Schaffung eines starken Beamtentums einhergegangen. Aber erst nach dem Siebenjährigen Krieg wurden im Rahmen eines „Rétablissements“ erste Schritte auf dem langen Weg zur Etablierung eines funktional gegliederten Beamtenapparats beschritten. Teil dieses Unterfanges war eine Reform der Beamtenausbildung, die wissenschaftlich gebildete und technisch kompetente Experten hervorbringen sollten. Erst Jahrzehnte später und nach einem weiteren Krieg fanden diese Bemühungen mit den SteinHardenbergschen Reformen einen vorläufigen Abschluss.

Im Mittelpunkt der hier vorgelegten historischen Rekonstruktion standen die Maßnahmen des Berliner Bergwerks- und Hüttendepartments um 1770 für eine Ausbildungsreform der Berg- und Hüttenbeamten sowie der Beamten für das Bau- und Forstwesen. Die Reform der Ausbildung von Berg- und Hüttenbeamten war ein besonders drängendes Problem für das 1768 geschaffene Bergwerks- und Hüttendepartment. Die revidierten preußischen Bergordnungen, die zwischen 1766 und 1772 verabschiedet wurden, folgten dem sächsischen Vorbild indem sie das „Direktionsprinzip“ einführten (Baumgärtel 1963:25-26, Müller-Erzbach 1916-17, 1: 95-99, Schulz-Briesen 1933: 28-29; Treue 1984). Das Direktionsprinzip unterstellte das gesamte Bergwesen, einschließlich der privaten Unternehmen, der staatlichen Leitung. ${ }^{94}$ Damit stieg der Bedarf an kompetenten Beamten dramatisch an, und zwar nicht nur der juristisch und fiskalisch versierten und an Universitäten ausgebildeten hohen 
Beamten, die für zentrale Verwaltungsaufgaben zuständig waren, sondern auch der mittleren und unteren Beamten, die lokale, betriebliche Leitungs- und technische Schlüsselfunktionen in den staatlich verwalteten Gruben und Hüttenwerken übernehmen konnten, wie Schichtmeister, Markscheider, Probierer, Schmelzer oder „Kunstmeister“ (später „Maschineningenieure“ oder ,-techniker").

Vor allem drei Kameralisten trieben die Reform der Beamtenausbildung aktiv voran, der Bergrat Carl Abraham Gerhard und die beiden Minister Ludwig Philipp Freiherr von Hagen und Carl Joseph Maximilian Freiherr von Fürst und Kupferberg. Aktive Beiträge leisteten auch der Akademiebotaniker und Dozent für Forstwirtschaft Johann Gottlieb Gleditsch, der Baurat und Dozent für Mathematik Friedrich Holsche und der Apotheker und Chemiker Valentin Rose, die ebenso wie Gerhard die Persona des hybriden wissenschaftlich-technischen Experten verkörperten. Die Debatten, die diese Reform begleiteten, beschäftigten sich zum einen mit der Frage finanzierbarer Institutionen und zum anderen mit dem Verhältnis von schulischem Wissen („Theorie“) und nachfolgender beruflicher Tätigkeit („Praxis“). Indirekt betrafen sie auch die Frage, was nützliche Wissenschaften seien. Es ging sowohl um die Frage des „Wie“ als auch des „Was“ des nützlichen wissenschaftlichen Unterrichts. Alle Akteure, die in diesem Aufsatz oft direkt zu Wort kamen, beabsichtigten eine Reform der preußischen Beamtenausbildung unter Einbeziehung der Wissenschaften, und alle strebten eine „Bergwerkswissenschaft" an. Hinter der um 1770 geführten Debatte über das Verhältnis von Theorie und Praxis verbarg sich also keine prinzipielle Skepsis über den Nutzen der Wissenschaften für das Berg- und Hüttenwesen oder die Bau- und Forstwirtschaft. Jedoch war das, was als nützliche Wissenschaft gelten sollte, nur ansatzweise vorhanden, denn es gab keine fertig entwickelte „Bergwerkswissenschaft", die bloß zu übernehmen und anzuwenden gewesen wäre.

Die beteiligten Akteure waren sich im Prinzip einig, dass es darum ging, verschiedenartige Wissensbestände zu integrieren, die von systematischem Wissen über die Natur bis zu mathematischem und technischem Expertenund Ingenieurwissen reichten. Große Unterschiede gab es jedoch hinsichtlich der Frage wie die "Theorie“, das heißt schulisches Wissen, mit der Praxis insbesondere der zukünftigen Berg- und Hüttenbeamten zu verbinden sei und wie sich dies im Unterricht niederschlagen sollte. Gerhard schlug eine gemeinsame schulische Ausbildung aller Berg- und Hüttenbeamten vor, der eine praktische, speziellere Ausbildung in Gruben oder Hüttenwerken nachfolgen sollte. Dieser Vorschlag ging mit Reformvorschlägen für den schulischen Unterricht selbst einher. Experimente, Modelle, Zeichnungen und ein Mineralienkabinett sollten Elemente der beruflichen Praxis in die schulische „Theorie“ hinein nehmen. Gerhards Vorschlag einer zeitlichen Trennung von „Theorie und Praxis“ stieß jedoch auf vehementen Widerspruch der 
zuständigen preußischen Minister, die sich in dieser Hinsicht an der Freiberger Akademie orientierten. Festzuhalten bleibt hier jedoch, dass es um 1800 auch andere einflussreiche Stimmen gab, die für eine einheitliche wissenschaftliche Ausbildung der etwa 16-jährigen Schüler argumentierten. So erhielten an der 1794 gegründeten Pariser École polytechnique alle Schüler dieselbe allgemeine wissenschaftliche Ausbildung bevor sie sich an einer der écoles d'application weiter spezialisierten (Belhoste 2003). Auch das 1821 in Berlin gegründete Königliche Gewerbeinstitut vermied eine frühe Spezialisierung und versuchte stattdessen, „vollständige theoretische Kenntnisse für alle Zweige“ des Gewerbes und alle Techniker zu vermitteln (Lundgren 1975: 61).

Ein Teil der hier rekonstruierten Aktivitäten ist in die Geschichte als Gründung der Berliner „Bergakademie“ eingegangen. Minister und leitende Kameralisten in den preußischen Behörden unternahmen um 1770 eine Reihe von Maßnahmen für die Förderung des wissenschaftlichen und technischen Unterrichts ihrer Beamten, aber eine Bergakademie wurde eindeutig nicht gegründet. Die Behauptung, 1770 sei eine Bergakademie oder „bergtechnische Hochschule" (Krusch 1904) in Berlin etabliert worden, konstruiert einen Gründungsmythos der späteren, erst 1860 eindeutig identifizierbaren Berliner Bergakademie, der diese wohl mit den Weihen Friedrichs „des Großen“ versehen sollte. Die Geschichte der wissenschaftlichen Ausbildung der preußischen Berg- und Hüttenbeamten im 18. Jahrhundert muss daher auch als eine Geschichte des Scheiterns erzählt werden. Dieses Scheitern ist in erheblichem Maße auf die Politik Friedrichs II. zurückzuführen. Es spricht vieles dafür, dass Friedrich II. die wissenschaftliche Ausbildung seiner Beamten weitgehend den preußischen Universitäten überlassen wollte, und zwar aus Kostengründen. Die Bereitschaft des "Philosophenkönigs", in Wissenschaften, Technik und Wirtschaft zu investieren, war insgesamt gering und stets schwankend. Sie musste ihm fast immer von reformfreudigen Ministern abgerungen werden. Ohne diese limitierenden politischen und finanziellen Faktoren wäre vermutlich einiges anders gelaufen. Dennoch waren die öffentlichen Vorlesungen des Bergwerks- und Hüttendepartments nicht wirkungslos. Sie fungierten als ein Rekrutierungsfeld für wissenschaftlich und technisch kompetente preußische Beamte und als eine Institution zur Verbreitung von Wissensformen, die anschlussfähig an neue gewerbliche Techniken waren. Trotz ihres prekären und instabilen institutionellen Status war 1770 eine Institution geschaffen worden, die die Produktion wissenschaftlich-technischen Wissens förderte, verbreitete und über ihre "Schüler" mit der gewerblichen Praxis in Verbindung brachte. 


\section{Danksagung}

Ich danke Johannes Lotze für seine wertvolle Hilfe bei meiner Archivarbeit und für seine sorgfältigen Transkriptionen. Hans-Jörg Rheinberger danke ich für die finanzielle Unterstützung meiner Archivarbeit.

\section{Anmerkungen}

1 „Generaldirektorium“ steht für „General-Ober-Finanz-Kriegs- und Domänendirektorium".

2 Preußen war in dieser Hinsicht kein Ausnahmefall. Siehe etwa Bartels 2010, Brianta 2000, Dym 2008, Lundgren 1975, Vaccari 2000, Vogel 2008, Wakefield 2009, Weber 1976. Aus Platzgründen kann hier und im Folgenden nur eine enge Auswahl der relevanten Sekundärliteratur zitiert werden. Für weitere Literatur zum Verhältnis von Wissenschaften und handwerklichen Künsten der Frühen Neuzeit verweise ich auf die umfangreiche Bibliographie in Klein/Spary 2010.

3 Geheimes Staatsarchiv Preußischer Kulturbesitz, I. HA, Rep. 121 Ministerium für Handel und Gewerbe, Abt. Bergwerks-, Hütten- und Salinenwesen (im Folgenden: GStA PK mit Aktennummer abgekürzt), Nr. 7957 (Acta betreffend den wissenschaftlichen Unterricht der Berg-, Hütten- und Salinen-Aspiranten. Einrichtung einer Bergakademie), Bl. 15.

4 Kurz vor dem Siebenjährigen Krieg dienten zwei Drittel der jährlichen Staatseinnahmen von ca. 13 Millionen Reichstalern zum Unterhalt der Armee (Treue 1984: 82). Die Kosten für den Siebenjährigen Krieg betrugen ca. 139 Millionen Taler. 1770 betrugen die jährlichen Einnahmen des preußischen Staates ca. 21,7 Millionen Reichstaler; davon wurden mindestens 13 für die Armee ausgegeben (Duffy 1974: 130-131).

5 Diese Behauptung geht maßgeblich auf Krusch 1904 zurück. Sie wurde insbesondere durch Strunz 1970, der Kruschs Forschungsergebnisse unkritisch übernahm, bekräftigt. Zweifel äußerte jedoch Vogel 2008: $136 \mathrm{f}$.

6 Zu Gerhard siehe Hufbauer 1982: 193-195, Lyncker 1934: 143.

7 „Naturforscher“ ist ein Terminus des 18. Jahrhunderts, „Geologie“ einer des späten 18. Jahrhunderts.

8 Ich verwende den Terminus technischer Experte als Sammelbegriff für Ingenieure und andere avancierte technische Sachverständige im weiteren Sinn, für die es im 18. Jahrhundert nur Einzelbezeichnungen gab, wie z.B. Apotheker, Kunstmeister, Feuerwerker oder „Bergwercks-Gelehrter“ und „Bau-Verständiger“ (Zimmermann 1769). Für die engere Bezeichnung „Techniker" siehe Lundgren 1975.

9 Zur Figur des ,guten“ Kameralisten siehe Wakefield 2009, zu der des hybriden, wissenschaftlich-technischen Experten Klein/Spary 2010; speziell zu Gerhard als wissenschaftlich-technischem Experten Klein, in Vorbereitung. Zu den hybriden, wissenschaftlich-technischen Experten des 18. Jahrhunderts gehörten auch die Apotheker-Chemiker; siehe dazu Klein 2007a, b.

10 Die meisten neueren Studien über die französische École polytechnique und écoles d'application haben ihr Hauptaugenmerk auf die Schüler, die späteren Ingenieure und Techniker, gelegt und weniger auf die Lehrer, von denen viele die Persona des hybriden, wissenschaftlich-techischen Experten verkörperten (z.B. der Gründer der École polytechnique Gaspar Monge); siehe z. B. Alder 1997. Picon 1992 (469-588) geht zwar auf Lehrer ein, analysiert aber nicht die Persona. Eine wichtige Ausnahme ist Belhoste 2003.

11 Die erste Anregung für die Gründung einer Bergschule, einschließlich eines Laboratoriums, in Berlin kam von Gerhard und dem Geheimen Finanzrat Heinrich Wilhelm Reichardt. Sie findet sich in einem Bericht an von Hagen über eine Inspektionsreise nach Schlesien vom 10. November 1769, siehe Fechner 1901: 249.

12 GStA PK Nr. 7957: Bl. 2-6.

13 Ebd.: Bl. 2, Hervorhebung U. K. 
Ebd.: Bl. 4, 5.

Ebd.: Bl. 2, Hervorhebung U. K.

Ebd.

$\mathrm{Zu}$ dieser Bedeutungsvariante des Begriffspaars Theorie und Praxis siehe auch Kant [1793] 1968.

GStA PK Nr. 7957: Bl. 2-3.

Ebd.: Bl. 2.

Ebd.: Bl. 3.

Ebd.; Bl. 2.

Ebd.: Bl. 2, 5.

Ebd.: Bl. 4.

Ebd.: Bl. 9.

Ebd.:Bl. 8.

Ebd.: Bl. 9.

Ebd.: Bl. 74. Von Hagen starb am 2. Februar 1771.

Gerhards Bericht vom 9. Februar 1770, siehe ebd.: Bl. 12-14.

Ebd.: Bl. 16.

Brief von Fürsts vom 4. März 1770, ebd.: Bl. 27-30.

Ebd.: Bl. 29.

Ebd.

Ebd.: Bl. 30.

Ebd. Bl. 37.

Ebd.: Bl. 10, 18-20.

Ebd.: Bl. 43.

Ebd.: Bl. 15.

Ebd.: Bl. $46 a$.

Ebd.: Bl. 42-43, Acta Borussica, Bd. 15: 280-293, Johnson 1975: 232 f.

GStA PK Nr. 7957: Bl. 42.

Ebd: Bl. 43, Hervorhebung U. K.

Von Hagens Initiative für die Ausbildungsreform der Baubeamten wurde durch von Fürsts „Reglement" für die Examina der Beamten des neuen Oberbaudepartments sowie für die des "Justiz Kollegiums“ und des „Finanz und Cameral-Wesens" (vom 29. Juni 1770) unterstützt (ebd: Bl. 65-69). Zu den erst später eingeführten Examina der Berg- und Hüttenbeamten siehe Zix 1911.

GStA PK Nr. 7957: Bl. 27.

Ebd.

Zu dieser Initiative von Hagens siehe ebd.: Bl. 17, 56. Im April und Mai 1770 wurden auch die schlesischen Gymnasien und Hauptschulen über die königliche Intention die „Bergwerkswissenschaften“ zu fördern unterrichtet; ebd., Bl. $44 \mathrm{f}$. Zu Realschulen und anderen technischen Schulen des 18. Jahrhunderts siehe Albrecht 1989.

GStA PK Nr. 7957: Bl. 27.

Ebd.: Bl. 28, Hervorhebung U. K.

Ebd., Hervorhebung U. K.

Ebd., Hervorhebung U. K.

Ebd.: Bl. 33.

Ebd., Hervorhebung U. K.

Die Anordung stammte vom 2. Januar 1766.

Ebd.: Bl. 42-43.

Ebd.: 43.

Ebd., Hervorhebung U. K.

Acta Borussica, Bd. 15: 282-291; GStA PK Nr. 7957: Bl. 43.

Zur politischen Praxis des ,aufgeklärten Absolutismus“ Friedrichs II. siehe Neugebauer 2009, 345-55.

GStA PK Nr. 7957: Bl. 34-35, Hervorhebung U. K.

Walter, auch Walther geschrieben, war seit 1760 zweiter Professor und ab 1774 erster Professor für Anatomie und Physik am Berliner Collegium medico-chirurgicum. Ab 1773 war er ordentliches Mitglied der Preußischen Akademie der Wissenschaften.

Ebd.: Bl. 28. 
61 Ebd.: Bl. 29.

62 Ebd.: Bl. 36.

63 In einem zusammenfassenden Bericht vom 22. Oktober 1777 bemerkte Baurat Holsche zu diesem geringen Gehalt: „Die Stiftung ist geschehen und es hat ein jeder Lehrer die festgesetzte $100 \mathrm{R}$ [eichsthaler] seit dieser Zeit erhalten; weil kein fernere Salarirung erfolget ist, so hat auch dieses Institut nur so vielen Nuzzen gehabt, als von einer solchen geringen Besoldung erwartet werden können." GStA PK Nr. 7958: Bl. 74, Hervorhebung U.K..

64 GStA PK Nr. 7957: Bl. 46.

65 Ebd.: Bl. 55.

66 Ebd.: Bl. 79.

67 Ebd.: Bl. 56.

68 Ebd.: Bl. 109.

69 Ebd.: Bl. 111, Hervorhebung U. K.

70 Hinzugefügt werden muss dennoch, dass auch der Terminus „Berg-Akademie“ 1774 für kurze Zeit verwendet wurde (GStA PK Nr. 7958: Bl. 48).

71 GStA PK Nr. 7957: Bl. 110.

72 Ebd.: Bl. 116, 122-123.

73 Die Bibliothek wurde bereits im März 1770 erwähnt (GStA PK Nr. 7957: Bl. 24).

74 GStA PK Nr. 141 (Acta betreffend das für das Bergwerks-Department einzurichtende und zu unterhaltende Laboratorium): Bl. 14-24.

75 GStA PK Nr. 7957: Bl. 162. Kurella war der Schwiegersohn des Akademiechemikers und ersten Professors für Chemie am Collegium medico-chirurgicum Johann Heinrich Pott, siehe Lehmann 1936: 59.

76 GStA PK Nr. 7957: Bl. 162, 179.

77 Ebd.: Bl. 140-41; GStA PK, Nr. 7958 (Acta betreffend den wissenschaftlichen Unterricht der Berg-, Hütten- und Salinen-Aspiranten, Bd. 2): Bl. 16-18.

78 GStA PK Nr. 7958: Bl. 7.

79 GStA PK Nr. 7957: Bl. 165-66.

80 Ebd.: Bl. 172.

81 GStA PK Nr. 7958: Bl. 2.

82 GStA PK Nr. 7957: Bl. 144.

83 Ebd.: Bl. 146.

84 Ebd.: Bl. 110.

85 Ebd.

86 Ebd.: Bl. 113-114.

87 Ebd.: Bl. 146.

88 Ebd.

89 Ebd., Hervorhebung U. K.

90 Ebd.: Bl. 160. Valentin Rose starb am 28. April 1771.

91 GStA PK Nr. 141: Bl.4, Bl. 8.

92 GStA PK Nr. 7957: Bl. 53. Zudem regte Gerhard an, auch Modelle von Öfen und Maschinen zu kaufen; darauf hin erhielt er die Auskunft, diese könnten aus den Registraturen ausgeliehen werden; GStA PK Nr. 7957: Bl. 55. Im Oktober 1775 legte Gerhard einen Bericht über seine Vorlesungen vor, der auch eine Liste von „Experimenta“ einschloss; demnach führte Gerhard auch ein „Journal“ über seine Experimente, ebd. Bl. 56-60.

93 GStA PK Nr. 7958: Bl. 16-21.

94 Eine Ausnahme waren die königlichen Salinen in Preußen, die bis Anfang der 1790er Jahre an private Unternehmer verpachtet und erst danach durch Salzämter verwaltet wurden; siehe Vogel 2008: 135-142. 


\section{Literaturverzeichnis}

Albrecht, Helmuth, 1989. Die Anfänge eines technischen Bildungssystems. In: Laetita Boehm und Charlotte Schönbeck, Hg., Technik und Bildung, Düsseldorf: VDI-Verlag, 118-153 [(Technik und Kultur Bd. V].

Alder, Ken, 1997. Engineering the Revolution. Arms and Enlightenment in France, 1763-1815, Princeton: Princeton Universty Press.

Bartels, Christoph, 2010. The Production of Silver, Copper, and Lead in the Harz Mountains from Late Medieval Times to the Onset of Industrialisation. In: Ursula Klein und Emma C. Spary, Hg., Materials and Expertise in Early Modern Europe. Between Market and Laboratory. Chicago/London: The University of Chicago Press, 71-100.

Baumgärtel, Hans, 1963. Bergbau und Absolutismus. Der sächsische Bergbau in der zweiten Hälfte des 18. Jahrhunderts und Maßnahmen zu seiner Verbesserung nach dem Siebenjährigen Kriege. Leipzig: VEB Deutscher Verlag für Grundstoffindustrie.

Baumgärtel, Hans, 1965. Vom Bergbüchlein zur Bergakademie. Zur Entstehung der Bergbauwissenschaften zwischen 1500 und 1765/1770. Leipzig: VEB Deutscher Verlag für Grundstoffindustrie.

Belhoste, Bruno, 2003. La formation d'une technocratie. l'école polytechique et ses élèves de la Révoltion aus Second Empire. Paris: Belin.

Brianta, Donata, 2000. Education and Training in the Mining Industry, 1750-1860: European Models and the Italian Case. Annals of Science, 57, 267-300.

Duffy, Christopher, 1974. The Army of Frederick the Great. Newton Abbot u. a.: David \& Charles.

Dym, Warren, 2008. Scholars and Miners. Drowsing and the Freiberg Mining Academy. Technology and Culture, 49, 833-859.

Fechner, Hermann, 1901. Geschichte des Schlesischen Berg- und Hüttenwesens in der Zeit Friedrich des Grossen, Friedrich Wilhelm's II. und Friedrich Wilhelm's III. 1741 bis 1806. Zeitschrift für das Berg-, Hütten- und Salinen-Wesen im Preussischen Staate, 49, 1-86, 243288, 383-446, 487-569.

Harnack, Adolf, 1900. Geschichte der Königlich Preussischen Akademie der Wissenschaften zu Berlin, 3 Bde. Berlin: Reichsdruckerei.

Herrmann, Walther, 1953. Bergbau und Kultur. Beiträge zur Geschichte des Freiberger Bergbaus und der Bergakademie. Berlin: Akademie Verlag.

Herrmann, Walther, 1962. Bergrat Henckel. Ein Wegbereiter der Bergakadiemie. Berlin: Akademie Verlag.

Hufbauer, Karl, 1982. The Formation of the German Chemical Community (1720-1795). Berkeley: University of California Press.

Johnson, Hubert C., 1975. Frederick the Great and his Officials. New Haven/London: Yale University Press.

Kahlow, Andreas, 2000. Die ersten Jahre der Berliner Bauakademie: Vorgeschichte und Zeitbild um 1800. In: Karl Scharz, Hg., 1799-1999. Von der Bauakademie zur Technischen Universität Berlin, Geschichte und Zukunft. Berlin: Ernst \& Sohn, 32-55.

Kant, Immanuel, [1793] 1968, Über den Gemeinspruch: Das mag in der Theorie richtig sein, taugt aber nicht für die Praxis. In Kants Werke, Akademie-Textausgabe Bd. 8, Berlin: Walter de Gruyter, 273-313.

Klein, Ursula, 2007a. Apothecary's Shops, Laboratories and Chemical Manufacture in EighteenthCentury Germany. In: Lissa Roberts, Simon Schaffer und Peter Dear, Hg., The Mindful Hand. Inquiry and Invention from the Late Renaissance to Early Industrialization. Amsterdam: Koninklijke Nederlandse Akademie van Wetenschappen, 247-276.

Klein, Ursula, 2007b. Apothecary-Chemists in Eighteenth-Century Germany. In: Lawrence M. Principe, Hg., New Narratives in Eighteenth-Century Chemistry. Dordrecht: Springer, 97137.

Klein, Ursula, in Vorbereitung. Carl Abraham Gerhard: Wissenschaftlich-technischer Experte und guter, preußischer Kameralist. In Peter Konecny und Hartmut Schleiff, Hg., Staat, Bergbau und Bergakademie im 18. und frühen 19. Jahrhundert.

Klein, Ursula/Spary, Emma C., Hg., 2010. Materials and Expertise in Early Modern Europe. Between Market and Laboratory. Chicago und London: The University of Chicago Press. 
Königliche Akademie der Wissenschaften, Hg., Acta Borussica: Denkmäler der preußischen Staatsverwaltung im 18. Jahrhundert, 1893-1982, Bd. 1-16.2, Berlin: Parey.

Krusch, Paul, 1904. Die Geschichte der Bergakademie zu Berlin von ihrer Gründung im Jahre 1770 bis zur Neueinrichtung im Jahre 1860. Berlin: Königliche Geologische Landesanstalt und Bergakademie.

Lehmann, Herbert, 1936. Das Collegium medico-chirurgicum in Berlin als Lehrstätte der Botanik und der Pharmazie. Berlin: Triltsch \& Huther.

Lundgren, Peter, 1975. Techniker in Preussen während der frühen Industrialisierung, Ausbildung und Berufsfeld einer entstehenden sozialen Gruppe, Berlin: Colloquium Verlag.

Lyncker, von Alexander, 1934. Die Matrikel des preußischen Collegium medico-chirurgicum in Berlin 1730 bis 1768. Archiv für Sippenforschung und alle verwandten Gebiete, 11, 5: 129-158.

Müller-Erzbach, Rudolf, 1916-17. Das Bergrecht Preußens und des weiteren Deutschlands. 2 Bde., Stuttgart: Ferndinand Enke.

Neugebauer, Wolfgang, 2009. Brandenburg-Preußen in der Frühen Neuzeit. Politik und Staatsbildung im 17. Und 18. Jahrhundert. In: Wolfgang Neugebauer, Hg., Handbuch der Preussischen Geschichte, Bd. 1, Berlin und New York: Walter de Gruyter, 113-407.

Nicolai, Friedrich, 1786. Beschreibung der Königlichen Residenzstädte Berlin und Potsdam, aller daselbst befindlicher Merkwürdigkeiten, und der umliegenden Gegend, 3. Auflage, Berlin: Friedrich Nicolai.

Picon, Antoine, 1992. L'invention de l'ingénieur moderne. L'Ecole des Ponts et Chaussées 17471851, Paris: Presses Ponts et chaussées.

Schlüter, Christoph Andreas, 1738. Gründlicher Unterricht von Hütte-Werken. Braunschweig: Meyer.

Schulz-Briesen, Max, 1933. Der preußische Staatsbergbau von seinen Anfängen bis zum Ende des 19. Jahrhunderts. Berlin: Reimar Hobbing.

Straubel, Rolf, 1998. Beamte und Personalpolitik im altpreußischen Staat. Soziale Rekrutierung, Karriereverläufe, Entscheidungsprozesse (1763/86-1806). Potsdam: Verlag für BerlinBrandenburg.

Strunz, Hugo, 1970. Von der Bergakademie zur Technischen Universität Berlin, 1770-1970. Berlin: Technische Universität Berlin.

Treue, Wilhelm, 1984. Wirtschafts- und Technikgeschichte Preussens. Berlin/New York: de Gruyter.

Vaccari Ezio, 2000. Mining and Knowledge of the Earth in Eighteenth-Century Italy. Annals of Science, 57, 163-180.

Vogel. Jakob, 2008. Ein schillerndes Kristall. Eine Wissensgeschichte des Salzes zwischen Früher Neuzeit und Moderne. Köln u. a.: Böhlau.

Wagenbreth, Otfried, 1994. Die Technische Universität Bergakademie Freiberg und ihre Geschichte, dargestellt in Tabellen und Bildern. Leipzig/Stuttgart: Deutscher Verlag für Grundstoffindustrie.

Wakefield, Andre, 2009. The Disordered Police State. German Cameralism as Science and Practice. Chicago/London: The University of Chicago Press.

Weber, Wolfhard, 1976. Innovationen im frühindustriellen Bergbau und Hüttenwesen. Friedrich Anton von Heynitz. Göttingen: Vandenhoeck \& Ruprecht.

Zimmermann, Carl Friedrich, [1744] 1769. Vorrede von den Bergwercks-Wissenschafften zu Vermehrung der Cammeral-Nutzungen. In: Johann Friedrich Henckel, Kleine Mineralogische und chymische Schriften, auf Gutbefinden des Herrn Autoris, nebst einer Vorrede von den Bergwercks-Wissenschafften zu Vermehrung der Cammeral-Nutzungen und mit Anmerkungen herausgegeben von Carl Friedrich Zimmermann, 2. Aufl., Wien/Leipzig: F. Jahn.

Zix, (Berginspektor), 1911. Die Ausbildung der höheren Staatsbeamten in Preußen (1778 bis 1897). Zeitschrift für das Berg-, Huetten- und Salinenwesen im Deutschen Reich, 59, 1-61.

Ursula Klein

Max-Planck-Institut für Wissenschaftsgeschichte

Boltzmannstraße 22

14195 Berlin

Deutschland 